\title{
Keeping Faith with Homoskedasticity or Going Back to Heteroskedasticity? The Case of Fatang Stocks
}

José Dias Curto ( $\square$ dias.curto@iscte-iul.pt )

ISCTE-Instituto Universitário de Lisboa: ISCTE-Instituto Universitario de Lisboa https://orcid.org/00000003-2012-9015

\section{Research Article}

Keywords: US stock markets, Volatility , Persistence , Half-life, Semikurtosis

Posted Date: March 10th, 2021

DOI: https://doi.org/10.21203/rs.3.rs-226549/v1

License: (c) (i) This work is licensed under a Creative Commons Attribution 4.0 International License. Read Full License 


\title{
KEEPING FAITH WITH HOMOSKEDASTICITY OR GOING BACK TO HETEROSKEDASTICITY? THE CASE OF FATANG STOCKS ${ }^{\star}$
}

\author{
José Dias Curto
}

Received: date / Accepted: date

\begin{abstract}
Did the pattern of US stock markets volatility change due to the COVID-19 or have the US stock markets been less volatile despite the pandemic shock? And as for tech stocks, are they even less volatile than the overall market? In this paper we provide evidence in favour of a "quietness" in the stock markets, interrupted by the COVID-19, by analysing dispersion, skewness and kurtosis characteristics of the empirical distribution of nine returns series including individual FATANG stocks (FAANG: Facebook, Amazon, Apple, Netflix and Google; plus Tesla) and US indices (S\&P500, DJIA and NASDAQ). A new methodology based on semi-variance and semi-kurtosis is proposed. Structural breaks and conditional heteroskedasticity are also analyzed by considering the traditional symmetrical and asymmetrical GARCH models. We show that in the most recent past, despite the COVID-19 pandemic, the FATANG tech stocks are characterized mostly by conditional homoskedasticity while the returns of US stock indices are mainly conditional heteroskedastic.
\end{abstract}

Keywords US stock markets · Volatility · Persistence · Half-life · Semikurtosis

\section{Introduction}

After the 2008 subprime mortgage crisis the US stocks and indices have started a notable recovery, giving rise to the longest bull market in US financial markets history. As one can see in Figure 1 the S\&P 500 achieved its minimum (676.53) in March 9, 2009 and from there it was almost always going up.

\footnotetext{
* This work was supported by Fundação para a Ciência e a Tecnologia, grant UID/GES/00315/2013.

Instituto Universitário de Lisboa (ISCTE-IUL), BRU-UNIDE, Lisboa, Portugal, Department of Quantitative Methods for Management and Economics,

Av. Prof. Aníbal Bettencourt, 1600-189 Lisboa, Portugal.

Phone: 35121 7826100. Fax: 35121 7938709, E-mail: dias.curto@iscte-iul.pt
} 
Fig. 1 S\&P 500 and VIX

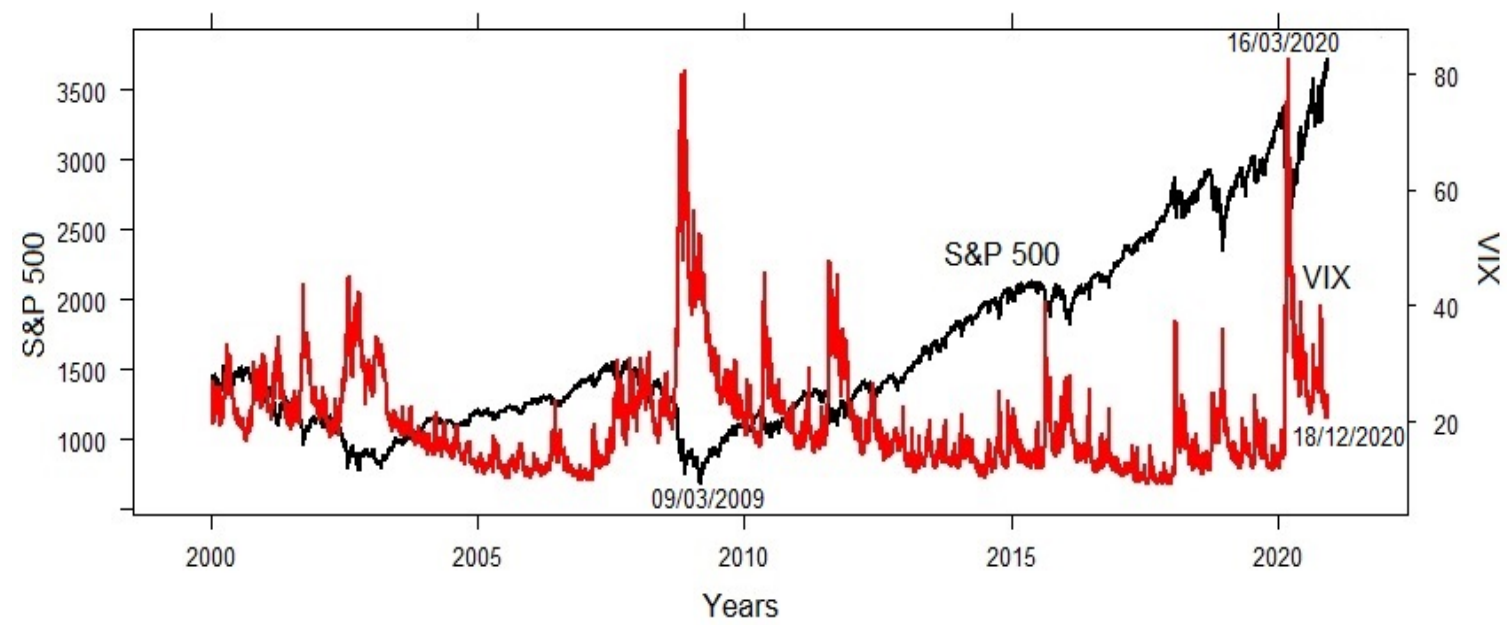

The stunning growth is evident from the numbers in Table 1 . The stock price of Netflix, for example, grew $9617.3 \%$ in 11 years giving rise to a remarkable $51.6 \%$ geometric average annual return. High growth rates characterizes also other US stocks and indices.

Table 1 Growth of US stocks and indices

\begin{tabular}{lrrrr} 
& \multicolumn{2}{c}{ Prices } & \multicolumn{2}{c}{ Growth } \\
Index/Stock & $09 / 03 / 2009$ & $18 / 12 / 2020$ & Total & Average \\
\hline S\&P 500 & 676.53 & 3709.41 & $448.3 \%$ & $16.7 \%$ \\
DJIA & 6547.05 & 30179.05 & $361.0 \%$ & $14.9 \%$ \\
NASDAQ & 1268.64 & 12755.64 & $905.5 \%$ & $23.3 \%$ \\
AMAZON & 60.49 & 3201.65 & $5192.9 \%$ & $43.4 \%$ \\
APPLE & 2.56 & 126.66 & $4847.7 \%$ & $42.6 \%$ \\
NETFLIX & 5.50 & 534.45 & $9617.3 \%$ & $51.6 \%$ \\
GOOGLE & 144.50 & 1731.01 & $1097.9 \%$ & $25.3 \%$ \\
\hline
\end{tabular}

And about volatility, what happened in the most recent past? Before 2020 there was a "quietness" in the stock markets occasionally interrupted by short stressful periods with increasing volatility. However, the market quickly recovered and returned to its upward trend. The Fed's predictable policy is one reason experts say stock-market volatility has been historically low. Over the past few years, Fed policy has been cautious and relatively predictable.

In 2018 and 2019, with the US economy humming along and interest rates no longer so close to zero, the case for strong forward guidance about future policy actions was becoming less compelling. According to John Williams, in his first speech as President of the New York Fed, it was not so clear as before 
whether interest rates should go up or down and explicit forward guidance, or promises about how the Fed will behave, would not be longer appropriate. Thus, it seemed that the future direction of policy would not be as clear as during the past few years, bringing more uncertainty and volatility to the stock markets ${ }^{1}$. Despite this, the US stocks and indices reached new historical highs at the end of 2019.

The year 2020, specially in March, due to the COVID-19 spread worldwide, and not because of a Fed policy change, was characterized by unusual variations in stock prices leading to a period of extreme high volatility. As one can see in figure 1, the Chicago Board Options Exchange (CBOE) Volatility Index (VIX), a popular measure of the stock market's expectation of volatility based on S\&P 500 index options, achieved its 82.69 highest value in March, 16. Recall that VIX values greater than 30 are commonly associated with high instability, while values below 20 compare to less distressing times in the financial markets.

After the intervention of Governments and Central Banks, with stimulus of billions of dollars and euros, the capital markets calmed down after March, 2020 (the VIX quickly returned to pre-COVID levels), which poses another million dollar question for financial analysts and investors: are these recent stable market conditions able to continue or can a new stressed regime of volatility be expected to arise in the near future? Are the severity and frequency of the swings seen on Wall Street last year (markets have not seen moves this wild since the subprime mortgage financial crisis) here to stay or are they just a brief period of turmoil and the calm will return soon with the economic recovery? Thus, the question "Keeping faith with homoskedasticity or going back to heteroskedastcity?" seems to make sense.

As early noted in the seminal papers of [29] and [15], financial time series vary systematically with time, showing periods of enormous unpredictability and volatility, trailed by times of low instability. Regardless of these early investigations, and the importance of modelling and forecasting volatility in financial markets (see, for instance, [30]), the efforts to model volatility dynamics have only been developed in the last decades of the 20th century. Until there it was assumed the errors' homoskedasticity in traditional econometric models, i.e., the focus of financial time series modelling was the conditional first moment, neglecting any temporal dependencies in the higher order moments.

The volatility of financial assets has been extensively studied for the last thirty years, since the seminal papers of [13] and [4] were published, and it remains a hot topic of investigation due to its importance for investors, financial analysts and academics. Volatility modelling, and especially volatility dynamics, are important for decision making in financial markets including derivative prices, bonds, leverage ratios, credit spreads and portfolio decisions. Recently, [52] investigates the effect of financial sector development on the growth volatility by using the data of 50 countries. The empirical results show that broadly speaking the aggregate growth volatility declines from 1997 to

1 See Robb, Greg at https://www.marketwatch.com/, September 28, 2018 for details. 
2014 while the most developed countries have much smaller growth volatility than the other countries. [41] investigated the determinants of six euro area sovereign bond yield spreads between 2016.06 and 2007.01, by estimating original Panel-GARCH models, incorporating key stylized features of volatility dynamics, such as extreme persistence, asymmetry [32] and risk premia effects. They found, in accordance to financial literature, significant and quite persistence on volatility, although the presence of asymmetric effects on the volatility process seems negligible. [42] analyzed volatility spillover effects in interbank money markets. Empirical evidence shows that money markets are profoundly interrelated, displaying dynamic cross market impacts. Besides, they highlight the pertinence of conditional covariances, showing that volatility spillovers are time-varying and strongly related to the major economic events, increasing during periods of higher uncertainty, which supports the need to closely monitoring the evolution of money markets.

Not so recent, but still focusing on volatility, [36] examined volatility spillovers between oil prices and emerging economies. Their significant results show the weak integration among the Chinese financial markets, energy markets and the US stock market. On the other hand, Brazilian, Indian and Russian markets were found to be more sensitive to international shocks resulting from US markets, and also to the instability of prices in the energy markets, particularly as to oil price uncertainty.

Due to changes in volatility, [40] examined the importance of structural breaks in exchange rates volatility by using in-sample and out-of-sample tests. The results point for the presence of structural breaks in the unconditional variance of seven out of eight US dollar exchange rate return series over the 1980-2005 period. This would suggest unstable results, with GARCH(1,1) estimates frequently shifting across subsamples resulting from the structural breaks, impacting particularly in the volatility persistence over time.

Structural breaks in volatility have also been tested and considered by [49], [8] and [53], among many others. Using daily data for six major international stock market indices and a modified EGARCH specification, [8] analyze the relation between stock market returns, volatility and trading volume by proposing a new nonlinear conditional variance model with multiple regimes and volume effects (MSV-EGARCH). By using the Harvey- Newbold test for multiple forecast encompassing, they show that the more complex MSV-EGARCH threshold structure dominates the competing standard asymmetric models (GJR and EGARCH) in terms of forecasting ability, for several of the considered stock indices. [49] compares the ability of traditional diagnostic tests to detect various sorts of breaks in GARCH models. The results show that robust LM tests proposed by [54] have no power to detect structural breaks in GARCH models. When CUSUM - and LM - based structural break tests are considered, the results point for an excellent size when the data is Gaussian. However, the CUSUM tests tend to over-reject in fat tails returns, no matter the sample size. The LM-based tests show approximately the correct size and the power is very high to detect various sorts of breaks in the dynamics of conditional volatility. [53] analyzed the interaction between 
oil and the US dollar exchange rate. The results point that ignoring structural breaks can increase the negative volatility correlation between the oil and USD exchange rate markets, with particular impact during the financial crisis. [47] studied the volatility of the Bitcoin cryptocurrency highlighting the importance of jumps and structural breaks in forecasting its volatility. In the out-of-sample analysis, they found that the HARQ-F-J model is the best model, showing the importance of the temporal variation and squared jump components at different time spans.

More recently, the COVID-19 triggered a number of new researches, in line with ours, to assess the impact of corona virus on the volatility of financial assets returns. By using an extended GARCH-MIDAS model and a newly developed Infectious Disease Equity Market Volatility Tracker (EMVID), [2] investigate the effects of COVID-19 on volatility of US, China, UK and Japan stock markets through January 2005 to April 2020. The empirical results show that, up to 24-month lag, infectious disease pandemic has significant positive impacts on the permanent volatility, even after controlling for several factors, namely the influence of past realized volatility, global economic policy uncertainty and the volatility leverage effect. [44] assesses the relevance of health-news trends in the predictability of stock returns. The results show that the model incorporating health-news index outperforms the benchmark historical average model, highlighting the importance of health news as a good predictor (due to its significance) of stock returns since the emergence of the pandemic. [46] found, based on the Asymmetric Power GARCH model, that COVID-19 has a substantial negative impact and reduces the US and Japan's market returns. Moreover, the influence of COVID-19 in the variance of the US, Germany, and Italy's stock markets is higher when compared to the Global Financial Crisis of 2008.

This small selection of papers illustrates how the volatility of financial asset returns remains a very important investigation topic in finance. Therefore, and due to the expected structural breaks in volatility, we want to address how the three main stylized facts of the volatility of returns [7]: clustering, persistence and asymmetry, have evolved (and changed) over time for the nine series under analysis. We focus mainly before and after the COVID-19 shock in 2020.

The remainder of this paper is organized as follows. In Section 2 we present the news and existent statistical and econometrical methods used in the paper. A new indicator based on semi-kurtosis is proposed to evaluate the downside risk. In Section 3 we give a brief description of the data we use: the daily prices and returns of twelve financial series, including stocks and indices. In Section 3 we discuss some properties of the empirical distribution of returns, namely dispersion, skewness and kurtosis; we also analyze the autocorrelation of returns, absolute returns and squared returns. Results confirm the stronger correlation when the absolute and the square returns are considered. Time varying volatility is modelled in Section 5 by using ARMA-GARCH (GARCH, GJR and EGARCH) specifications. Before estimating the models, we first tested for (potentially multiple) structural breaks in the unconditional variance of daily returns. Inspection of the estimated models across the sub-samples 
defined by the structural breaks point for differences in parameter estimates, volatility clustering, volatility persistence and asymmetric effect. Section 6 concludes the analysis and sheds some light on the volatility of returns.

\section{The methods}

To answer the question "Keeping faith with homoskedasticity or going back to heteroskedastcity?" we start by computing descriptive statistics (mean, standard deviation, skewness and kurtosis) over a rolling window encompassing the previous year of daily observations $(T=250)$, to notice the temporal changes. ANOVA, Kruskal-Wallis and Levene tests are used to compare means, distributions and variances of returns between different periods. A simple linear regression is also estimated to conclude about the linear relation between means and variances of returns (see Subsections 4.1 and 4.2).

A new methodology based on semi-variance and semi-kurtosis is proposed and described next (see Subsection 2.1) to compare the two sides of the mean and to distinguish between risk and uncertainty. The empirical results appear in Subsection 4.2.

Existent econometric methodologies are also used. To conclude with regard to the autocorrelation of returns, absolute returns and square returns over time, the Ljung-Box (LB) test for up to the tenth order serial correlation is computed over the rolling window process. Lagrange Multiplier test [13] is used to formally test the presence of conditional heteroskedasticity and the evidence of ARCH effects. An ARMA(4,0) model is estimated firstly to pre-filter the data from linear dependency. See Subsection 4.3.

The econometrical approach is detailed next in Subsection 2.2 and empirical results appear in Section 5.

\subsection{Semi-variance and semi-kurtosis}

In the Subsection 4.2 we show that, when the individual FATANG stocks are considered, the estimates of skewness can be either positive or negative and the peaks of kurtosis are also explained by large positive returns leading to the positive spikes of the coefficient of skewness. Thus, large positive returns also inflate the kurtosis in the case of the FATANG stocks. This empirical evidence has important consequences in terms of risk analysis. Variance (as a measure of volatility) and kurtosis increase mainly due to large negative returns (indices) and also to large positive returns (stocks), but only the former represent an increase in risk, because the chance of selling the asset at a lower price (than its purchase price) is higher. If an asset receives a large positive return, the event is considered an increase in uncertainty but not in risk. Both large negative and positive returns produce an increase in variance and kurtosis, because they take equally into account movements in either tail of the distribution, but only those on the left side are undesirable [19]. Thus, it is important to 
separate the "bad" from "good" volatility and kurtosis, because the former represents risk while the latter only represents uncertainty.

Let $\sigma^{2-}=\sum_{t=1}^{T_{1}}\left(r_{t}-\mu\right)^{2-}$ and $K^{-}=\sum_{t=1}^{T_{1}}\left(r_{t}-\mu\right)^{4-}$ represent the downside of the variance and the coefficient of kurtosis, respectively: all the observations that fall below the mean. If we divide $\sigma^{2-}$ and $K^{-}$by $T$ and $T \sigma^{4}$, respectively, we get the semi-variance [31] and the semi-kurtosis (see [43] for an application). We can also define the upside as $\sigma^{2+}=\sum_{t=1}^{T_{2}}\left(r_{t}-\mu\right)^{2+}$ and $K^{+}=\sum_{t=1}^{T_{2}}\left(r_{t}-\mu\right)^{4+}$, including all the observations that fall above the mean. With,

$$
\begin{gathered}
\sum_{t=1}^{T_{1}}\left(r_{t}-\mu\right)^{2-}+\sum_{t=1}^{T_{2}}\left(r_{t}-\mu\right)^{2+}=\sum_{t=1}^{T}\left(r_{t}-\mu\right)^{2}, \\
\sum_{t=1}^{T_{1}}\left(r_{t}-\mu\right)^{4-}+\sum_{t=1}^{T_{2}}\left(r_{t}-\mu\right)^{4+}=\sum_{t=1}^{T}\left(r_{t}-\mu\right)^{4}, \quad \text { with } T=T_{1}+T_{2},
\end{gathered}
$$

obviously being the numerators of the variance and the coefficient of kurtosis, respectively.

In order to evaluate the downside risk, we can divide the semi-variance by the variance and the semi-kurtosis by the kurtosis, respectively, given rise to the ratios:

$$
R_{V}=\frac{\sigma^{2-}}{\sigma^{2}}=\frac{\sum_{t=1}^{T_{1}}\left(r_{t}-\mu\right)^{2-}}{\sum_{t=1}^{T}\left(r_{t}-\mu\right)^{2}}, \quad R_{K}=\frac{K^{-}}{K}=\frac{\sum_{t=1}^{T_{1}}\left(r_{t}-\mu\right)^{4-}}{\sum_{t=1}^{T}\left(r_{t}-\mu\right)^{4}},
$$

and both measures range between 0 and 1 (the extremes are not included) and the risk decreases when the value of the ratios goes to zero (for values lower than 0.5 , the upside "dominates" the downside). If the value is 0.5 , the two sides are balanced and the distribution is symmetric. The risk increases when the value of the ratios is higher than 0.5 (the downside "dominates" the upside).

\subsection{Econometrical approach}

The empirical distribution of a financial asset return can be described as the sum of a predictable part with an unpredictable part:

$$
r_{t}=E\left[r_{t} \mid \Phi_{t-1}\right]+u_{t},
$$

where $\Phi_{t-1}$ is the relevant information set until, and including, $t-1$. For the conditional mean, $E\left[r_{t} \mid \Phi_{t-1}\right]$, our first intuition was to assume a white noise process, since the empirical distributions of returns under study represent the most liquid and efficient financial markets in the world - as far equities are concerned - and since this work is primarily dedicated to the dynamics of the variance equation. However, anticipating our findings in the data analysis section, we shall also specify the conditional mean equation as a fourth-order 
autoregressive process, $\mathrm{AR}(4)$, in order to remove the observed linear dependency in returns:

$$
r_{t}=c+\phi_{1} r_{t-1}+\phi_{2} r_{t-2}+\phi_{3} r_{t-3}+\phi_{4} r_{t-4}+u_{t},
$$

where $u_{t}=z_{t} \sigma_{t}$ and the standardized innovations $\left(z_{t}\right)$ are assumed to be independently and identically distributed (i.i.d.) with Student's $t$ distribution [5]. This statistical distribution has a long tradition in the econometrics literature as a popular choice of a fat-tailed distribution, since it has finite second moment (in contrast to stable non-Gaussian distributions), its mathematical properties are well known, it is undemanding to estimate, and is often found capable of capturing the excess of kurtosis observed in financial time-series. Other non-Normal alternative distributions have also been used in econometrical literature. [37] proposed the Generalized Error distribution (GED), the Laplace distribution has been employed in [18] and [22] used both the Student's $t$ and GED as distributional alternative models for innovations. The stable Paretian distributions have been also investigated by [28], [35] and [9].

For the conditional variance of $u_{t}: E\left[u_{t}^{2} \mid \Phi_{t-1}\right]=\sigma_{t}^{2}$, we have considered the most popular conditional heteroskedastic specifications: the symmetric GARCH [4] and the asymmetric GJR [17] and EGARCH [37] models to incorporate the leverage effect (see for example [50] and [10]). As observed by [3], volatility responds asymmetrically to the sign of any change in the price of the financial asset, i.e., volatility increases more after negative changes than after positive changes of the same magnitude. This phenomenon has become known as the leverage effect (also referred as the Fisher-Black effect).

In this study, with the estimation of asymmetrical models, we want to test if the leverage effect is also a characteristic of FATANG stocks. We show that in some periods of time positive and negative news have the same impact on volatility; having no asymmetrical effect. However, the leverage effect is still present in all the stock indices analyzed.

Despite the theoretical interest of $(p, q)$ models, the $(1,1)$ specification is, in general, satisfactory when modeling financial assets returns volatility (see [6] and more recently [20]). Thus, in this paper all conditional heteroskedastic models are of $p=1, q=1$ order:

$$
\begin{gathered}
\text { GARCH: } \quad \sigma_{t}^{2}=\omega+\alpha_{1} u_{t-1}^{2}+\beta_{1} \sigma_{t-1}^{2}, \\
\text { EGARCH: } \quad \ln \sigma_{t}^{2}=\omega+\alpha_{1} \frac{\left|u_{t-1}\right|}{\sigma_{t-1}}+\gamma_{1} \frac{u_{t-1}}{\sigma_{t-1}}+\beta_{1} \ln \sigma_{t-1}^{2}, \\
\text { GJR: } \quad \sigma_{t}^{2}=\omega+\alpha_{1} u_{t-1}^{2}+\gamma_{1} I_{t-1} u_{t-1}^{2}+\beta_{1} \sigma_{t-1}^{2},
\end{gathered}
$$

where $\omega, \alpha_{1}, \gamma_{1}$ and $\beta_{1}$ are unknown parameters, $I_{t-1}=1$ if $u_{t-1}<0$ and $I_{t-1}=0$ if $u_{t-1} \geq 0$. The models are estimated through maximum likelihood (MLE). Estimated models are compared based on Bayesian Information Criteria [48].

Several studies show that structural breaks have potentially important for estimating GARCH models of the volatility of returns, and neglecting structural breaks has important consequences. First, the degree of persistence in 
the volatility of returns can be overstated ([24], [33] and [21]). Secondly, [33], [34] and [38] show that structural breaks can give rise to spurious evidence of long-range dependence or long memory in financial volatility data. Thirdly, in out-of-sample volatility forecasting, the use of an expanding data window (or a fixed data window) is unlikely to perform well in the presence of sudden structural breaks in volatility [40]. Thus, before estimating GARCH, GJR and EGARCH models we test firstly for the existence of structural breaks in the unconditional variance of returns of the nine series under analysis, which according to [40] they are equivalent to structural breaks in the parameters of the GARCH processes governing the conditional volatility of returns.

To identify structural breaks in the volatility of the nine returns series, we apply a modified version of the [23] iterated cumulative sum of squares (ICSS) algorithm that allows for dependent processes proposed by [40]. The algorithm is used to test for (potentially multiple) structural breaks in the unconditional variance of daily returns for the nine series under study. For more details about the ICSS algorithm, see also [45], [26], [11] and [27], for example. The PELT algorithm [25] was also used and the results were similar. The advantage of PELT is that it can be used directly through the changepoint $R$ package. Thus, the samples are partitioned in distinct periods.

In this study, testing for the presence of structural breaks in volatility has two main objectives. Firstly, did COVID-19 result in a structural break for all the returns series or just for some? We show that no changes on volatility have occurred for some of the FATANG stocks, pointing for conditional homoskedasticity. Secondly, we want to analyze, how the three stylized facts of the returns' volatility [1]: clustering, persistence and asymmetry, have evolved over time, and if the differences are statistically significant.

\section{The data}

The data sets we will analyze in this paper are the daily closing prices and the continuously compound returns of the three main US stock indices: S\&P500, DJIA and NASDAQ and six American stocks, five under the acronym FAANG, referring to the stocks of the five most popular and best-performing American technology companies: Facebook, Amazon, Apple, Netflix and Alphabet (formerly known as Google). In this paper we also include Tesla and the acronym is changed to FATANG. The starting date is not common to all series (see Table 2 for details), but we try to cover as long a period as possible. For example, in the case of FATANG stocks, prices go back to the first day each company has been listed in the New York Stock Exchange (except APPLE). The end date is December 18, 2020.

We analyze the continuously compounded percentage rates of return (adjusted for dividends) that are calculated by taking the first differences of the logarithm of the series $\left(P_{t}\right.$ is the closing value for each index or stock at time $t)$ :

$$
r_{t}=100 \times\left[\ln \left(P_{t}\right)-\ln \left(P_{t-1}\right)\right] .
$$


Table 2 summarizes the basic statistical properties of the data. All the results, with the exception of skewness (which is positive for some series), comply with the stylized facts of returns.

Table 2 Summary statistics of $r_{t}$

\begin{tabular}{lrrrrrrrrrr} 
Index/Stock & Starting date & \# Obs & Mean & Median & Min & Max & St Dev & Skew & Kurt & J-B \\
\hline S\&P 500 & $29 / 01 / 1985$ & 9047 & 0.033 & 0.063 & -22.900 & 10.957 & 1.163 & -1.252 & 29.937 & 0.000 \\
DJIA & $30 / 01 / 1985$ & 9047 & 0.035 & 0.058 & -25.632 & 10.764 & 1.148 & -1.596 & 41.728 & 0.000 \\
NASDAQ & $31 / 01 / 1985$ & 9047 & 0.042 & 0.113 & -13.149 & 13.255 & 1.397 & -0.345 & 11.809 & 0.000 \\
FACEBOOK & $18 / 05 / 2012$ & 2161 & 0.092 & 0.106 & -21.024 & 25.937 & 2.342 & 0.335 & 18.058 & 0.000 \\
AMAZON & $15 / 05 / 1997$ & 5939 & 0.125 & 0.049 & -28.457 & 29.618 & 3.650 & 0.455 & 11.941 & 0.000 \\
TESLA & $29 / 06 / 2010$ & 2637 & 0.189 & 0.116 & -23.652 & 21.829 & 3.535 & -0.033 & 9.039 & 0.000 \\
APPLE & $29 / 01 / 1985$ & 9047 & 0.078 & 0.009 & -73.125 & 28.689 & 2.834 & -1.985 & 59.105 & 0.000 \\
NETFLIX & $23 / 05 / 2002$ & 4677 & 0.130 & 0.035 & -52.605 & 35.223 & 3.638 & -0.876 & 26.289 & 0.000 \\
GOOGLE & $19 / 08 / 2004$ & 4113 & 0.086 & 0.069 & -12.340 & 18.225 & 1.915 & 0.453 & 12.130 & 0.000 \\
\hline Skew: Coeff. of Skewness, Kurt: Coeff. of Kurtosis and J-B is the $p$-value associated to the & & & &
\end{tabular}

The means of return are all positive but close to zero (the higher means correspond to the FATANG stocks). The distribution of returns appears to be somewhat asymmetric as reflected by negative and positive skewness estimates. All the series returns have heavy tails and show a strong departure from normality (skewness and kurtosis coefficients ${ }^{2}$ are all statistically different from those of the Normal distribution which are 0 and 3, respectively). The Jarque-Bera normality test statistic is far beyond the critical value (the $p$-value is almost zero) which suggests that $r_{t}$ is far from a normal distribution for all series.

Figure 2 plots the two most popular volatility measures regarding the financial assets return: the absolute value and the square of returns $\left(\left|r_{t}\right|\right.$ and $\left.r_{t}^{2}\right)$ [19]. We can observe the long run behavior of daily $\left|r_{t}\right|$ and $r^{2}$, where we can clearly confirm the observation of [29] and [15] that large shocks in financial asset returns tend to be followed by large shocks (of either sign) and small shocks tend to be followed by small shocks.

Market volatility is changing over time and it was much higher during the Black Monday stock market crash of 1987, the Russian ruble financial crisis in 1998, the dot-com bubble, where the Nasdaq Composite index peaked in value on March 10, 2000 before crashing, the United States subprime mortgage crisis, occurring between 2007 and 2010, and the COVID-19 pandemic in 2020.

In the recent months, and after the March COVID-19 turmoil, we have been observing a long period of quiet volatility, especially in the American stock markets. Is this just a cooling-off period for volatility or are the financial asset returns going back to homoskedasticity? To answer this question is one of the main purposes of this investigation.

2 From now on we just refer skewness and kurtosis. 
Fig. 2 Daily absolute returns
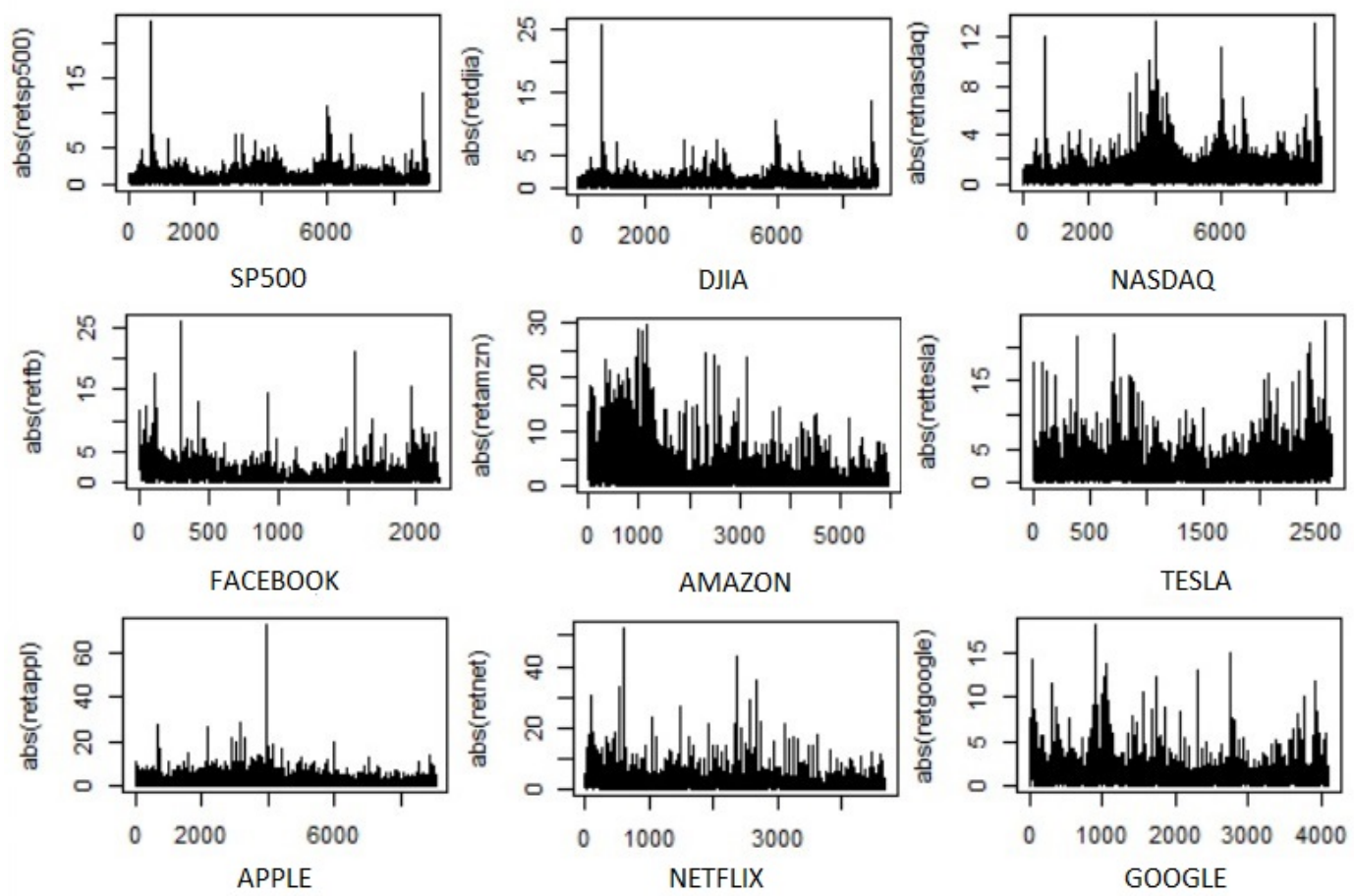

\section{Characteristics of the historical returns}

The measures presented in table 2 refer to the whole period under analysis. However, our expectation is that some characteristics of the empirical distributions will have evolved (and changed) over time, especially after the United States subprime mortgage crisis and the COVID-19 pandemic. To notice the temporal changes, all the measures are computed over a rolling window encompassing the previous year of daily observations $(T=250)$. Our first investigation topic relates to volatility, skewness and kurtosis of the empirical distribution of returns.

\subsection{Mean and standard deviation}

Have the stock markets been profitable and less volatile in recent years? And what happened to the tech stocks, are they even more profitable and less volatile than the overall market? To answer these questions we compute the arithmetic average and the standard deviation (due to the rolling window process, the first value is computed based on the first 250 observations over 
Fig. 3 Daily squared returns
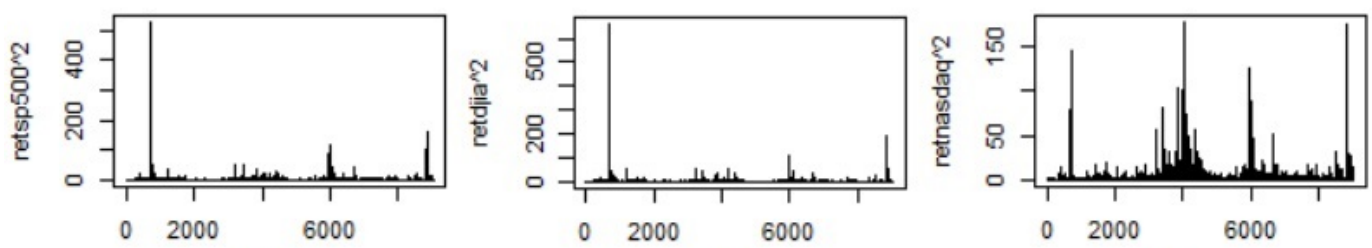

SP500
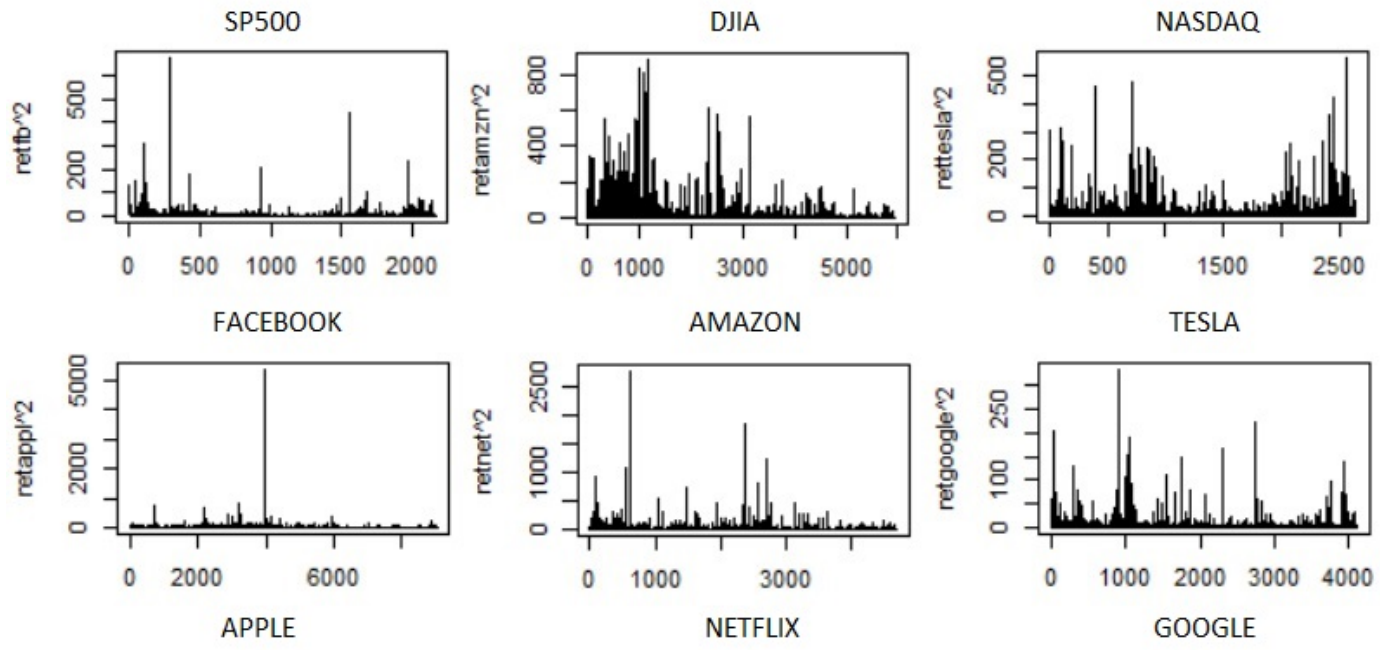

approximately one year and the last value, corresponding to December 18, 2020, is the average and variance of returns in 2020). See Figures 4 and 5.

As one can see, the daily average return is mostly positive (reflecting the prices increasing trend) but close to zero for all the series under analysis and higher for FATANG stocks. Due to the larger weight of these stocks in the composition of the index, the average return of NASDAQ is also higher when compared to S\&P 500 and DJIA. From 2012-2019 to 2020 there is an increase in the average return (except in the Dow Jones Industrial Average) - the ratio between the average returns is higher than 1, pointing for a temporal increase in 2020. However, the differences in the averages and distributions (before and after 2020) are not statistically significant - the exception is Tesla, where the daily average return was close to a stunning $0.864 \%$ in 2020 (see Table 3 for ANOVA and Kruskal-Wallis tests result). Thus, in spite of the COVID-19 pandemic, there was an increase (not statistically significant - the exception is Tesla) in the daily average return for 8 of the 9 series under scrutiny. The daily average return was 6.48, 2.58 and 2.34 times higher for Tesla, Apple and NASDAQ, respectively. The exception is the DJIA, where no tech stocks are included. The other two stock indices (NASDAQ and S\&P 500) also reflect 
the good performance of FATANG and other tech stocks during the pandemic period.

Table 3 Averages comparison before 2012, between 2012 and 2019 and after 2020

\begin{tabular}{lrrrrrrc} 
& Dec/2011 & $2012-2019$ & Ratio & 2020 & Ratio & ANOVA & K-W \\
\hline S\&P 500 & 0.029 & 0.047 & 1.634 & 0.056 & 1.202 & $0.469(0.494)$ & $3.896(0.143)$ \\
DJIA & 0.033 & 0.042 & 1.275 & 0.023 & 0.541 & $0.026(0.872)$ & $1.033(0.597)$ \\
NASDAQ & 0.033 & 0.061 & 1.860 & 0.144 & 2.336 & $1.726(0.189)$ & $9.361(0.009)$ \\
FACEBOOK & 0.113 & 0.075 & 0.664 & 0.121 & 1.625 & $0.009(0.923)$ & $0.361(0.835)$ \\
AMAZON & 0.122 & 0.118 & 0.967 & 0.224 & 1.906 & $0.047(0.828)$ & $0.687(0.158)$ \\
TESLA & 0.048 & 0.133 & 2.800 & 0.864 & 6.477 & $6.229(0.013)$ & $8.314(0.016)$ \\
APPLE & 0.070 & 0.088 & 1.246 & 0.226 & 2.580 & $0.448(0.503)$ & $2.675(0.263)$ \\
NETFLIX & 0.087 & 0.173 & 1.983 & 0.205 & 1.182 & $0.686(0.408)$ & $0.765(0.682)$ \\
GOOGLE & 0.100 & 0.071 & 0.707 & 0.105 & 1.486 & $0.085(0.770)$ & $2.262(0.323)$ \\
\hline
\end{tabular}

$\mathrm{K}-\mathrm{W}$ : Kruskal-Wallis test ( $p$-value is in parenthesis). Ratio is the division of average return between two periods. For example, the Ratio after column "2020" divides the average return for the year 2020 by the daily average return of the period 2012-2019. The columns "Dec/2011", "2012-2019" and "2020" include the daily average return in each period.

As expected, the peaks of volatility (measured by the standard deviation) occur during the most recent crises in the financial markets: 1987 Black Monday crash, 1998 Russian rubble financial crisis, 2000 dot-com bubble, 2008 subprime mortgage crisis and 2020 COVID-19 pandemic (see Figures 4 and $5)$. We can also observe a sharp decrease in volatility during the years 20122019 regardless of the index or the stock being considered. The results of table 4 confirm that the variance of returns decreases sharply from 2012 to 2019 and it increases significantly in $2020^{3}$ (the $p$-value associated with the Levene test $^{4}$ is in parenthesis, indicating the rejection of the equality of variances). In 2020 the increase in the variance of returns is higher for the three stock indexes (DJIA, for example, the variance is 8.848 times higher than its variance during the period 2012-2019) when compared to the individual stocks. In case of Netflix there is even a decrease in the variance of returns when compared to the variance of the period before. These results show that the increase in volatility was more pronounced in the three stock indexes when compared to the individual FATANG stocks. Furthermore, as the increase in the variance has been matched by a corresponding increase in the mean, we can conclude that positive returns more than compensate the negative ones. Thus, the increase in volatility does not necessarily imply a higher risk, as we explain next.

\footnotetext{
3 As Facebook has only been listed in the New York Stock Exchange after 2012, the cut-off year is 2015 .

4 With regard to departures from normality, the Levene test is less sensitive than the Bartlett test.
} 
Table 4 Variances comparison before 2012, between 2012 and 2019 and after 2020

\begin{tabular}{lrrrrrr} 
& Until Dec/2011 & $2012-2019$ & Ratio & 2020 & Ratio & \multicolumn{1}{c}{ Levene test } \\
\hline S\&P 500 & 1.431 & 0.655 & 0.457 & 4.925 & $\mathbf{7 . 5 2 5}$ & $109.08(0.000)$ \\
DJIA & 1.365 & 0.635 & 0.465 & 5.616 & $\mathbf{8 . 8 4 8}$ & $128.39(0.000)$ \\
NASDAQ & 2.135 & 0.938 & 0.439 & 5.248 & $\mathbf{5 . 5 9 8}$ & $84.59(0.000)$ \\
FACEBOOK & 8.737 & 3.193 & 0.365 & 8.606 & 2.695 & $61.66(0.000)$ \\
AMAZON & 19.202 & 3.459 & $\mathbf{0 . 1 8 0}$ & 5.985 & 1.730 & $243.99(0.000)$ \\
TESLA & 13.910 & 9.749 & 0.701 & 32.523 & 3.336 & $63.98(0.000)$ \\
APPLE & 9.611 & 2.604 & 0.271 & 8.830 & 3.391 & $178.23(0.000)$ \\
NETFLIX & 17.029 & 9.239 & 0.543 & 8.649 & $\mathbf{0 . 9 3 6}$ & $37.78(0.000)$ \\
GOOGLE & 5.033 & 2.123 & 0.422 & 6.021 & 2.836 & $79.67(0.000)$ \\
\hline
\end{tabular}

Ratio is the division of the variance from two different periods. For example, the Ratio after column "2020" divides the returns' variance of the year 2020 by the variance of the period 2012-2019. The columns "Until Dec/2011", "2012-2019" and "2020" include the variance for each period. Levene test $p$-value is in parenthesis.

Due to these strong differences in volatility, in section 5 we tested for the existence of structural breaks in the unconditional variance of the returns series and the results point to significant breaks.

Next we analyze also the relation between the mean and the variance of returns (per rolling window) in the three periods considered before by estimating the simple linear regression model: $\mu_{t}=\alpha+\beta \sigma_{r t}^{2}+\varepsilon_{t}$, where the dependent variable is the mean and the independent is the variance. Table 5 shows the estimates for $\beta$.

Table 5 Mean-Variance regression

\begin{tabular}{lrrr} 
Index/Stock & Until Dec/2011 & $2012-2019$ & 2020 \\
\hline S\&P 500 & $-0.0285^{*}$ & $-0.0365^{*}$ & $-0.0058^{*}$ \\
DJIA & $-0.0298^{*}$ & $-0.0473^{*}$ & $-0.0071^{*}$ \\
NASDAQ & $-0.025^{*}$ & $-0.0449^{*}$ & $\mathbf{0 . 0 0 7 6}$ \\
FACEBOOK & -0.0028 & $-0.0295^{*}$ & $\mathbf{0 . 0 0 8 8 ^ { * }}$ \\
AMAZON & $-0.0014^{*}$ & $-0.0248^{*}$ & $\mathbf{0 . 0 5 1 6}$ \\
TESLA & $\mathbf{0 . 0 4 1 4}^{*}$ & $\mathbf{0 . 0 2 6 6}$ & $\mathbf{0 . 0 3 0 1}$ \\
APPLE & $-0.0119^{*}$ & $-0.0685^{*}$ & 0.0016 \\
NETFLIX & $-0.0081^{*}$ & $-0.0131^{*}$ & $\mathbf{0 . 0 5 3 6}$ \\
GOOGLE & $-0.0258^{*}$ & $-0.0137^{*}$ & -0.0004 \\
\hline
\end{tabular}

Estimates for $\bar{\beta}$ in the simple linear regression model: $\mu_{t}=\alpha+\beta \sigma_{r t}^{2}+\varepsilon_{t}$, where $\mu_{t}$ and $\sigma_{r t}^{2}$ represent the rolling window mean and variance. ${ }^{*}$ the estimate is statistically significant at $1 \%$ significance level, based on the $p$-value associated to the corresponding $t$ significance test.

As one can see, the estimates are mostly negative until 2019 (the exception is Tesla). In 2020 most of estimates are positive ( 5 in 8 ), the estimates are not statistically significant ${ }^{5}$ in two cases (Apple and Google) and two estimates are negative (the ones of S\&P 500 and DJIA stock indices). Thus, for most of FATANG stocks ( 4 in 6 ) the average of returns increases with the variance; more

5 Due to potential autocorrelation, Newey-West HAC standard errors are used. 
volatility seems to indicate greater profitability for most of financial assets under analysis, highlighting the balance between positive and negative returns with supremacy of the first ones. Welcome volatility in US stock markets!

\subsection{Skewness and kurtosis}

Another common stylized fact of financial assets is that large negative returns occur more often than equally large upward movements, leading to the negative asymmetry of the empirical distributions [7]. However, in the recent past large positive returns have also occurred, reversing the type of asymmetry, especially regarding the FATANG stocks. In order to test this empirical fact, a rolling window encompassing the previous year's daily returns is used to estimate the coefficients of skewness and kurtosis. The results are shown graphically in Figures 6 and 7 .

As one can see, when the stock indices are considered, most of the estimates of skewness are negative and just a few are statistically significant and positive. Furthermore, the peaks in the kurtosis are mostly due to large negative returns, giving rise to the negative spikes of the coefficient of skewness. Thus, the number of large negative returns exceed those of positive returns, leading to the negative skewness of the empirical distributions. The conclusions are different when the individual FATANG stocks are analyzed. The estimates of skewness can be either positive or negative and the peaks of kurtosis are also explained by large positive returns leading to the positive spikes of the coefficient of skewness. Thus, large positive returns also inflate the kurtosis in the case of the FATANG stocks.

The ratio (percentage) between the semi-kurtosis and the kurtosis (see Subsection 2.1), computed based on the 250-day rolling window method, is represented graphically in Figures 8 and 9). The horizontal line represents $50 \%$.

As one can see, the value of the ratio (see Table 6) is always higher than $70 \%$ for the three American stock indices. Thus, the downside "dominates" the upside in more than $70 \%$ of the rolling windows. In case of stocks, it is the opposite, thus highlighting the importance of large positive returns when compared to the negative ones. Whereas the volatility and kurtosis of Facebook, Amazon, Netflix and Google stock returns represent more uncertainty than risk, they represent more risk than uncertainty in the case of stock indices. Thus, the indices seem to be riskier when compared to this particular class of tech stocks (in case of Tesla and Apple the ratio between semi-kurtosis and kurtosis is still higher than $50 \%$ in more that $50 \%$ of the rolling windows, but less then 20 percentage points when compared to the US stock indices). Thus, the chance to sell at a lower price than their purchase price seems to be higher in the case of stock indices. 
Table 6 Number of ratios above 50\%

\begin{tabular}{lrrc} 
Indice/Stock & \# ratio $i 50 \%$ & \# obs & $\%$ \\
\hline S\&P 500 & 6608 & 9047 & $73.04 \%$ \\
DJIA & 6630 & 9047 & $73.28 \%$ \\
NASDAQ & 6990 & 9047 & $77.26 \%$ \\
FACEBOOK & 986 & 2161 & $45.63 \%$ \\
AMAZON & 2350 & 5939 & $39.57 \%$ \\
TESLA & 1477 & 2637 & $56.01 \%$ \\
APPLE & 5132 & 9047 & $56.73 \%$ \\
NETFLIX & 2138 & 4677 & $45.71 \%$ \\
GOOGLE & 1671 & 4113 & $40.63 \%$
\end{tabular}

\subsection{Autocorrelation analysis of the return series}

It is well established that serial correlation (or autocorrelation) of financial asset returns is often insignificant ([16], [51] and [19]) giving empirical support to the weak-form of the Efficient Market Hypothesis (EMH), which states that past returns cannot be used to predict future returns. Thus, the returns are nearly white noise [19]. To conclude with regard to the autocorrelation of returns, absolute returns and square returns over time, the Ljung-Box (LB) test for up to the tenth order serial correlation is computed over a rolling window encompassing the previous year's daily observations $(T=250)$. The evolution of the LB test value is represented graphically in Figures 10, 11 and 12. The horizontal line represents the critical value for a $1 \%$ significance level: $\chi_{10}^{2}=23.20925$.

As one can see, and according to the Ljung-Box statistic for returns, the autocorrelation seems not to be relevant (in only a small percentage of windows is the "no autocorrelation" null hypothesis rejected, the exception being the NASDAQ composite index with more than $20 \%$ of rejections; see Table 7 where the number of rejections and the respective percentage are shown). The autocorrelation structure of returns still seems weaker in the case of individual stocks (in case of Tesla, for example, the number of rejections is very close to zero). In general, the rejection of the null hypothesis is due to the statistical significance of the positive first order and negative second order autocorrelations (this supports the so-called mean-reversion behaviour of stock market returns). In spite of its significance, the absolute value of the first and second order autocorrelations is very small, suggesting that returns $\left(r_{t}\right)$ do have some memory, albeit very short, and there is a portion of stock market returns that is predictable although it might be a very small one. So the efficient market or random walk hypothesis does not strictly hold [12].

Even though the series of returns seem to be weakly correlated over time, the autocorrelation of absolute and squared returns is stronger, pointing to a positive autocorrelation over several days, which quantifies the fact that high volatility events tend to cluster in time [7]. However, the volatility clustering stylized fact is not so evident for individual stocks, especially in the cases of Facebook, Amazon and Netflix. The empirical results seem also to confirm 
Table 7 Number of rejections of the null hypothesis in the Ljung-Box test

\begin{tabular}{l|rr|rr|rr} 
& \multicolumn{2}{|c|}{$r_{t}$} & & $|r t|$ & & $r_{t}^{2}$ \\
Indices/Stocks & \# Rejections & $\%$ & \# Rejections & $\%$ & \# Rejections & $\%$ \\
\hline S\&P 500 & 1158 & $13.16 \%$ & 4070 & $46.27 \%$ & 4105 & $46.66 \%$ \\
DJIA & 1188 & $13.50 \%$ & 4726 & $53.72 \%$ & 4220 & $47.97 \%$ \\
NASDAQ & 2138 & $24.30 \%$ & 5127 & $58.28 \%$ & 5209 & $59.21 \%$ \\
FACEBOOK & 259 & $13.55 \%$ & 736 & $38.51 \%$ & 396 & $20.72 \%$ \\
AMAZON & 199 & $3.50 \%$ & 1500 & $26.37 \%$ & 1194 & $20.99 \%$ \\
TESLA & 13 & $0.54 \%$ & 832 & $34.86 \%$ & 590 & $24.72 \%$ \\
APPLE & 657 & $7.47 \%$ & 2241 & $25.47 \%$ & 2052 & $23.33 \%$ \\
NETFLIX & 280 & $6.32 \%$ & 872 & $19.70 \%$ & 598 & $13.51 \%$ \\
GOOGLE & 426 & $11.03 \%$ & 1309 & $33.89 \%$ & 1043 & $27.00 \%$ \\
\hline
\end{tabular}

that sample autocorrelations for absolute returns are greater than the sample autocorrelations for squared returns.

Conditional heteroskedasticity of returns is a possible explanation for the large positive autocorrelation between $\left|r_{t}\right|$ and $r_{t}^{2}$, i.e., the variance or conditional variance is not constant and it is changing over time. ARCH type models is the most important class of nonlinear time series models that is able to capture some aspects of the time varying volatility structure. The Lagrange Multiplier test proposed by [13] can be used to formally test the presence of conditional heteroskedasticity and the evidence of ARCH effects. The LM test for a twelve-order linear ARCH effect is computed over a rolling window encompassing the previous year's daily observations $(T=250)$. An $\operatorname{ARMA}(4,0)$ model is estimated firstly to pre-filter the data from linear dependency. Figures 13 and 14 show the temporal evolution of the ARCH LM test for indices and stocks. The horizontal line represents the critical value for $1 \%$ significance level: $\chi_{12}^{2}=26.22$.

The ARCH-LM test results suggests, as it is common in empirical finance, that all the returns series under analysis exhibit ARCH effects, inferring that nonlinearities should enter through the variance of the processes [22]. ARCH or GARCH models can be used to capture such behavior, by conditioning the volatility of the process on past information. In section 5 we use ARMAGARCH models to describe the conditional distribution of returns. However, it seems that the conditional heteroskedasticity is more evident for stock indices and the $\mathrm{ARCH}$ effect has declined in the most recent past (the exception is the year 2020), especially in case of FATANG stocks. Thus, in the most recent past the market volatility is not changing so sharply over time and hence the question in the title 'Keeping faith with homoskedasticity or going back to heteroskedastcity?". In order to check this, we first computed the number of 250-day windows where the null hypothesis of the ARCH-LM test is rejected. Results are shown in table 8.

As one can see, the percentage of rejections is less than $20 \%$ in case of FATANG stocks and around $40 \%$ in case of US stock indices. Thus, the returns of US stock indices are more conditional heteroskedastic while the FATANG tech stocks returns are characterized mostly by conditional homoskedasticity. 
Table 8 ARCH-LM test: number of rejections

\begin{tabular}{lrrr} 
Indices/Stocks & \# Rejections & \# obs & $\%$ \\
\hline \&P 500 & 3360 & 8797 & $38.19 \%$ \\
DJIA & 3592 & 8797 & $40.83 \%$ \\
NASDAQ & 3864 & 8797 & $43.92 \%$ \\
FACEBOOK & 354 & 1911 & $18.52 \%$ \\
AMAZON & 982 & 5689 & $17.26 \%$ \\
TESLA & 303 & 2387 & $12.69 \%$ \\
APPLE & 1729 & 8797 & $19.65 \%$ \\
NETFLIX & 375 & 4427 & $8.47 \%$ \\
GOOGLE & 713 & 3863 & $18.46 \%$ \\
\hline
\end{tabular}

In both cases the percentage of rejections is lower than $50 \%$ : the number of nonrejections of conditional homoskedasticity assumption exceeds the number of rejections. However, this raises a new million dollar question: after the COVID19 turmoil, how long will it remain like this?

\section{Volatility clustering, persistence and asymmetry}

As we mentioned previously, our main purpose is to reach conclusions concerning the characteristics of volatility over a long period (focusing mainly on the last years) comparing FATANG stocks with three US stock indices. As the international financial markets are intermittently subject to shocks resulting from global economics, wars, political events and investors decisions, these shocks can cause sudden breaks in the unconditional variance of returns which are equivalent to structural breaks in the parameters of the returns conditionally volatility processes [40].

To identify and determine the structural breaks in the volatility of the nine returns series, we compute a modified version of the [23] iterated cumulative sum of squares (ICSS) algorithm that allows for conditional dependent processes proposed by [40]. The PELT algorithm [25] is also used and the results are similar. The number of each observation where each break occur and the exact dates of the structural breaks are reported in Table 9 .

The variances (Var in the table) are computed for each of the sub-samples defined by (and until) the structural breaks identified by the modified ICSS algorithm. As one can see, there is a strong evidence of structural breaks in the unconditional variance for all the returns series, leading to distinct regimes in volatility. The ICSS algorithm selects six structural breaks in the unconditional variance for the S\&P 500 and DJIA returns; five structural breaks for NASDAQ and Amazon; four structural breaks for Apple and Google and three structural breaks for Facebook, Netflix and Tesla. The unconditional variance in the sub-sample resulting from the penultimate structural break (in bold) is smaller when compared to the variance of most of the other sub-samples. This also confirms the smaller volatility in the years before February 2020, as we have pointed before. The variance increases sharply in the last sub-sample (the exception is Netflix, where it even occurs a decrease in variance) influenced 
Table 9 Structural breaks in the unconditional variance

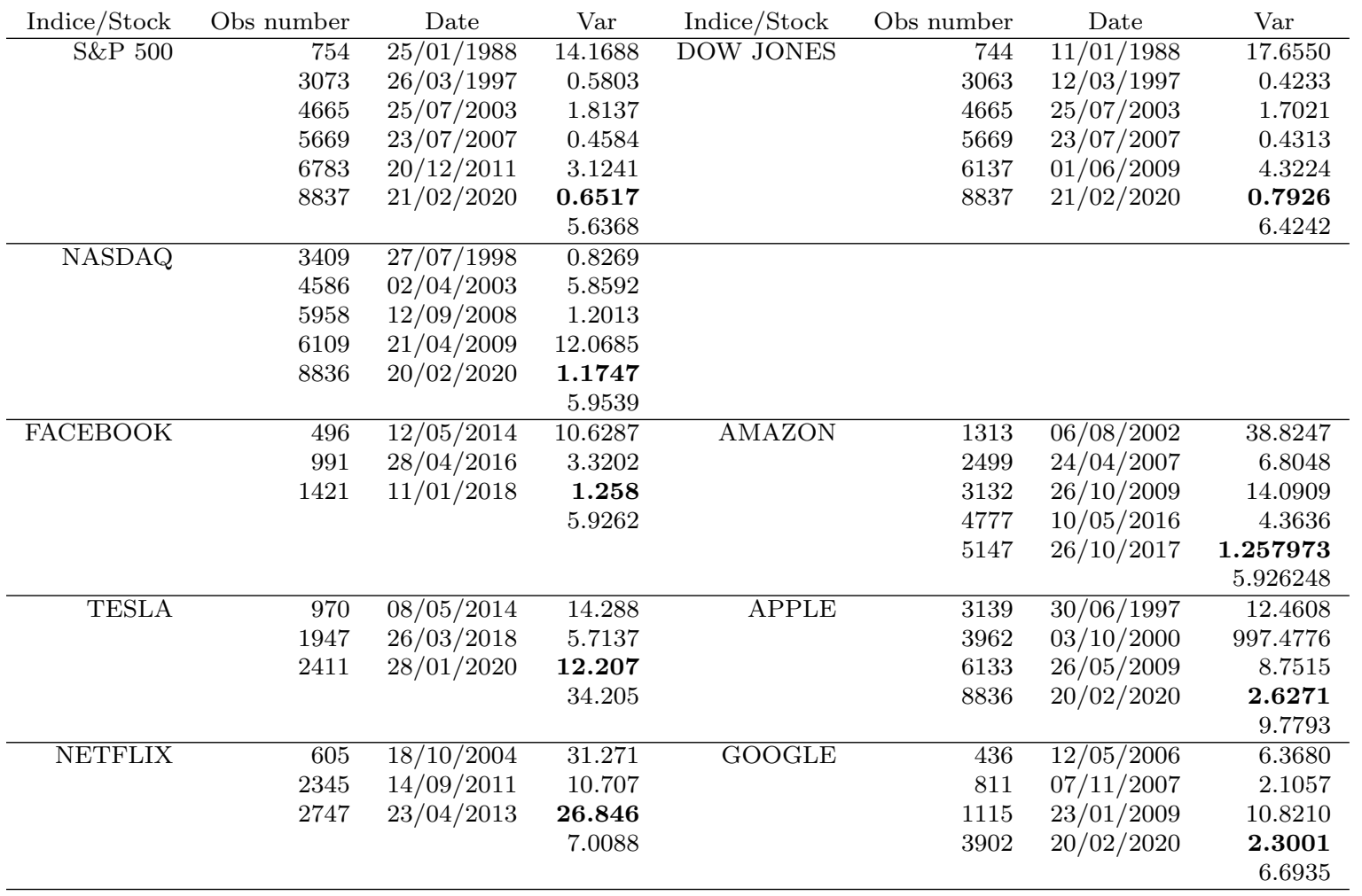

specially by the higher volatility of 2020. COVID-19 gave rise (in February 2020 ) to a new volatility regime in S\&P 500, DJIA, NASDAQ, Apple, Google and Tesla, but the increase in volatility was not enough to change the regime in the returns volatility of Amazon, Facebook and Netflix. It seems that $50 \%$ of the FATANG stocks were immune to the virus. Thus, we can question again: "Keeping faith with homoskedasticity or going back to heteroskedastcity?" Even for the six financial assets with structural break in volatility, was COVID just an accident and they will return quickly to the previous regime of almost constant variance?

Next we analyze the conditional distribution of returns to further clarify about volatility. We estimate an $\operatorname{ARMA}(4,0)$ model for the conditional mean in combination with $\operatorname{GARCH}(1,1)$, GJR(1,1) and $\operatorname{EGARCH}(1,1)$ models for the conditional variance across the sub-samples resulting from the structural breaks identified by the modified ICSS algorithm. To better account for leptokurtosis we consider the Student's $t$ distribution for innovations instead of the standardized normal. The estimation results across the sub-samples are shown in Tables 10 through 18. The analysis of results will be divided according to the three main stylized facts of the volatility of returns: volatility clustering, persistence and asymmetric effect. 
Table 10 GARCH, GJR and EGARCH(1,1), Persistence and Half-life - S\&P 500

\begin{tabular}{|c|c|c|c|c|c|c|c|c|}
\hline & Full sample & $25 / 01 / 1988$ & 26/03/1997 & $25 / 07 / 2003$ & $23 / 07 / 2007$ & $20 / 12 / 2011$ & $21 / 02 / 2020$ & $18 / 12 / 2020$ \\
\hline ARCH-LM & $980.282^{*}$ & 15.224 & $51.079^{*}$ & $107.330^{*}$ & $35.930^{*}$ & $335.662^{*}$ & $250.550^{*}$ & $56.268^{*}$ \\
\hline$\alpha$ & $0.093^{*}$ & -0.081 & $0.026^{*}$ & $0.076^{*}$ & -0.031 & $0.104^{*}$ & $0.191^{*}$ & $0.172^{*}$ \\
\hline$\beta$ & $0.902^{*}$ & 1.080 & $0.969 *$ & $0.888^{*}$ & 0.008 & $0.895^{*}$ & $0.774^{*}$ & $0.819^{*}$ \\
\hline$\alpha+\beta$ & 0.995 & 0.999 & 0.995 & 0.964 & NA & 0.999 & 0.965 & 0.991 \\
\hline Half-life & 130.76 & 692.801 & 138.283 & 18.905 & NA & 692.801 & 19.654 & 76.704 \\
\hline t-df & $5.508^{*}$ & 9.795* & $5.227^{*}$ & $9.242^{*}$ & $8.530^{*}$ & $6.601^{*}$ & $5.067^{*}$ & $5.810^{*}$ \\
\hline$\gamma$ GJR & $0.141 * *$ & $0.109 * * *$ & $0.018^{* * *}$ & $0.175^{*}$ & $0.159^{*}$ & $0.172^{*}$ & $0.367^{*}$ & 0.024 \\
\hline$\gamma$ EGARCH & $-0.108^{*}$ & $-0.142^{* *}$ & $-0.029 *$ & $-0.141^{*}$ & $-0.127^{*}$ & $-0.149^{*}$ & $-0.253^{*}$ & -0.048 \\
\hline BIC GARCH & 2.606 & 5.079 & 2.159 & 3.352 & 2.098 & 3.526 & 2.168 & 3.839 \\
\hline BIC GJR & 2.590 & 5.166 & 2.160 & 3.313 & 2.058 & 3.495 & 2.122 & 3.865 \\
\hline BIC EGARCH & 2.585 & 5.165 & 2.159 & 3.308 & 2.056 & 3.496 & 2.110 & 3.875 \\
\hline
\end{tabular}

ARCH-LM test. $*, * *, * * *$ denote statistically significant at the $1 \%, 5 \%$ and $10 \%$ significance levels, respectively. $\alpha+\beta=1$ is the measure of volatility persistence. Half-life gives the point estimate of the half-life in days given as $H L=\frac{\log (0.5)}{\log (\alpha+\beta)}$. $t$-df represents the Student's $t$ degrees of freedom. GARCH, GJR and EGARCH are conditional heteroskedastic models defined in (4), (6) and (5), respectively.

Table 11 GARCH, GJR and EGARCH(1,1), Persistence and Half-life - DJIA

\begin{tabular}{|c|c|c|c|c|c|c|c|c|c|}
\hline & Full sample & $11 / 01 / 88$ & $30 / 12 / 91$ & $12 / 03 / 97$ & $25 / 07 / 03$ & $23 / 07 / 07$ & 01/06/09 & $21 / 02 / 2020$ & $18 / 12 / 2020$ \\
\hline ARCH-LM & $751.758^{*}$ & $33.710^{*}$ & 6.607 & $28.331^{*}$ & $119.160^{*}$ & $27.039^{*}$ & $149.504^{*}$ & $419.496^{*}$ & $66.738^{*}$ \\
\hline$\alpha$ & 0.091* & $0.035 *$ & -0.018 & $0.046^{*}$ & $0.070^{*}$ & $0.043 * *$ & $0.090 *$ & $0.164^{*}$ & $0.213^{*}$ \\
\hline$\beta$ & $0.900^{*}$ & $0.937^{*}$ & $1.017^{*}$ & $0.914^{*}$ & $0.890^{*}$ & $0.910^{*}$ & $0.898^{*}$ & $0.819^{*}$ & $0.785^{*}$ \\
\hline$\alpha+\beta$ & 0.992 & 0.972 & 0.999 & 0.960 & 0.960 & 0.953 & 0.988 & 0.984 & 0.997 \\
\hline Half-life & 85.630 & 24.407 & 692.801 & 16.98 & 16.98 & 14.398 & 57.415 & 42.418 & 272.224 \\
\hline t-df & $5.555^{*}$ & $3.919 *$ & $6.622^{*}$ & $7.578^{*}$ & $8.44^{*}$ & $9.908^{*}$ & $21.077^{*}$ & $5.056^{*}$ & $8.447^{*}$ \\
\hline$\gamma$ GJR & $0.125 *$ & 0.022 & 0.028 & $0.914^{*}$ & $0.134^{*}$ & $0.168^{*}$ & $0.148^{*}$ & $0.305^{*}$ & 0.056 \\
\hline$\gamma \mathrm{EGARCH}$ & $-0.097^{*}$ & -0.025 & $-0.056^{* *}$ & $-0.077^{*}$ & $-0.113^{* *}$ & $-0.151^{*}$ & $-0.124^{*}$ & $-0.207^{*}$ & -0.055 \\
\hline BIC GARCH & 2.589 & 2.731 & 2.789 & 1.968 & 3.269 & 2.029 & 3.987 & 2.309 & 3.969 \\
\hline BIC GJR & 2.576 & 2.736 & 2.804 & 1.970 & 3.248 & 2.003 & 3.961 & 2.268 & 3.993 \\
\hline BIC EGARCH & 2.571 & 2.735 & 2.803 & 1.969 & 3.236 & 1.997 & 3.960 & 2.266 & 4.000 \\
\hline
\end{tabular}

ARCH-LM test. $*$, **, *** denote statistically significant at the $1 \%, 5 \%$ and $10 \%$ significance levels, respectively. $\alpha+\beta=1$ is the measure of volatility persistence. Half-life gives the point estimate of the half-life in days given as $H L=\frac{\log (0.5)}{\log (\alpha+\beta)} \cdot \mathrm{t}-\mathrm{df}$ represents the Student's $t$ degrees of freedom. GARCH, GJR and EGARCH are conditional heteroskedastic models defined in (4), (6) and (5), respectively.

We focus firstly on the ARCH-LM test results. As can be seen, we reject the null hypothesis of the ARCH-LM test, indicating the existence of conditional heteroskedasticity in most of the series and for most of the sub-samples resulting from the structural breaks. This conclusion confirms the stylized fact of the returns volatility clustering. However, among the FATANG stocks the decision is not the same for all the sub-samples. The stock returns of Facebook, Netflix (in all the sub-samples) Amazon, Apple, Tesla and Google (in some of the sub-samples) are characterized by conditional homoskedasticity. Furthermore, in case of Google and Tesla, and for recent years, conditional homoskedasticity was rejected only in the sub-sample that includes the COVID-19 turmoil. Before that, and for long periods of times, the null of the ARCH-LM test was not rejected. Thus, the suspicion of constant variance of returns is more evident among the FATANG stocks, questioning the existence of volatility clustering. 
Table 12 GARCH, GJR and EGARCH(1,1), Persistence and Half-life - NASDAQ

\begin{tabular}{|c|c|c|c|c|c|c|c|}
\hline & Full sample & $27 / 07 / 1998$ & $02 / 04 / 2003$ & $12 / 09 / 2008$ & $21 / 04 / 2009$ & $20 / 02 / 2020$ & $18 / 12 / 2020$ \\
\hline ARCH-LM & $2046.298^{*}$ & $1013.827^{*}$ & $116.000^{*}$ & $108.560^{*}$ & $33.232^{*}$ & $391.490^{*}$ & $40.722^{*}$ \\
\hline$\alpha$ & $0.111^{*}$ & $0.115^{*}$ & $0.096^{*}$ & $0.038^{*}$ & $-0.090 * * *$ & $0.131^{*}$ & $0.172^{* *}$ \\
\hline$\beta$ & $0.889^{*}$ & $0.860^{*}$ & $0.866^{*}$ & $0.956^{*}$ & $-0.605^{* *}$ & $0.846^{*}$ & $0.828^{*}$ \\
\hline$\alpha+\beta$ & 0.999 & 0.975 & 0.962 & 0.994 & -0.695 & 0.977 & 1.000 \\
\hline Half-life & 830.765 & 27.378 & 17.892 & 115.178 & NA & 29.802 & 1571.416 \\
\hline t-df & $6.726^{*}$ & $5.588^{*}$ & $34.853^{*}$ & $18.935^{*}$ & $89.826^{*}$ & $5.247^{*}$ & $3.891^{*}$ \\
\hline$\gamma \mathrm{GJR}$ & $0.120^{*}$ & $0.104^{*}$ & $0.178^{*}$ & $0.061^{*}$ & 0.229 & $0.303^{*}$ & $0.272^{*}$ \\
\hline$\gamma$ EGARCH & $-0.087^{*}$ & $-0.079^{*}$ & $-0.125^{*}$ & $-0.050^{*}$ & $-0.316^{* * *}$ & $-0.226^{*}$ & -0.122 \\
\hline BIC GARCH & 2.909 & 2.234 & 4.527 & 2.975 & 5.508 & 2.763 & 4.157 \\
\hline BIC GJR & 2.900 & 2.231 & 4.496 & 2.964 & 5.557 & 2.723 & 4.156 \\
\hline BIC EGARCH & 2.897 & 2.227 & 4.502 & 2.965 & 5.539 & 2.715 & 4.175 \\
\hline
\end{tabular}

ARCH-LM test. $* * *, * * *$ denote statistically significant at the $1 \%, 5 \%$ and $10 \%$ significance levels, respectively. $\alpha+\beta=1$ is the measure of volatility persistence. Half-life gives the point estimate of the half-life in days given as $H L=\frac{\log (0.5)}{\log (\alpha+\beta)}$. t-df represents the Student's $t$ degrees of freedom. GARCH, GJR and EGARCH are conditional heteroskedastic models defined in (4),

(6) and (5), respectively.

And what about volatility persistence? According to [14], in a $\operatorname{GARCH}(1$, 1) model, there are two main consequences if $\alpha+\beta=1$ : persistence on the conditional variance forecasting; and the variance of the unconditional distribution of the error term $\varepsilon_{t}$ is infinite. In other words, when $\alpha+\beta=1$ current shock persist indefinitely by conditioning the future variance. Thus, and according to [39], a significant impact of volatility on stock prices can only take place if shocks to volatility persist over a long time.

Table 13 GARCH, GJR and EGARCH(1,1), Persistence and Half-life - FACEBOOK

\begin{tabular}{lrrrrrr} 
& Full sample & $12 / 05 / 2014$ & $28 / 04 / 2016$ & $11 / 01 / 2018$ & $20 / 02 / 2020$ & $18 / 12 / 2020$ \\
\hline ARCH-LM & $20.50^{* *}$ & 0.685 & 14.647 & 15.829 & 15.921 & 12.811 \\
$\alpha$ & $0.045^{*}$ & 0.006 & $0.156^{* *}$ & $0.151^{* * *}$ & $0.112^{* * *}$ & $0.185^{*}$ \\
$\beta$ & $0.950^{*}$ & $0.979^{*}$ & 0.051 & 0.386 & 0.624 & $0.681^{*}$ \\
$\alpha+\beta$ & $\mathbf{0 . 9 9 6}$ & $\mathbf{0 . 9 8 5}$ & $\mathbf{0 . 2 0 7}$ & $\mathbf{0 . 5 3 7}$ & $\mathbf{0 . 7 3 6}$ & $\mathbf{0 . 8 6 7}$ \\
Half-life & 157.008 & 45.862 & 0.440 & 1.115 & 2.259 & 4.853 \\
t-df & $3.597^{*}$ & $4.338^{*}$ & $4.938^{*}$ & $4.070^{*}$ & 3.945 & $3.494^{*}$ \\
$\gamma$ GJR & $0.054^{*}$ & 0.036 & 0.086 & $0.256^{* *}$ & $0.209^{*}$ & $0.285^{*}$ \\
$\gamma$ EGARCH & $-0.043^{*}$ & -0.017 & -0.029 & $-0.134^{* *}$ & $-0.121^{*}$ & $-0.088^{*}$ \\
BIC GARCH & 4.123 & $\mathbf{5 . 0 2 0}$ & $\mathbf{3 . 9 5 7}$ & 3.074 & 3.083 & 4.346 \\
BIC GJR & 4.118 & 5.023 & 3.969 & 3.078 & 3.085 & 4.340 \\
BIC EGARCH & $\mathbf{4 . 1 1 0}$ & 5.033 & 3.971 & $\mathbf{3 . 0 7 3}$ & $\mathbf{3 . 0 7 9}$ & $\mathbf{4 . 3 3 9}$ \\
\hline
\end{tabular}

ARCH-LM test. $*, * *, * * *$ denote statistically significant at the $1 \%, 5 \%$ and $10 \%$ significance levels, respectively. $\alpha+\beta=1$ is the measure of volatility persistence. Half-life gives the point estimate of the half-life in days given as $H L=\frac{\log (0.5)}{\log (\alpha+\beta)} \cdot t-d f$ represents the Student's $t$ degrees of freedom. GARCH, GJR and EGARCH are conditional heteroskedastic models defined in (4), (6) and (5), respectively.

The parameter estimates resulting from the different models reveals that the $\operatorname{GARCH}(1,1)$ processes are highly persistent (almost-integrated) when the full sample is considered, with the $\alpha+\beta$ estimate ranging from 0.989 to 1.000 , in line with the extant literature. However, the persistence in the full-sample 
Table 14 GARCH, GJR and EGARCH(1,1), Persistence and Half-life - AMAZON

\begin{tabular}{|c|c|c|c|c|c|c|c|}
\hline & Full sample & $06 / 08 / 2002$ & $24 / 04 / 2007$ & $26 / 10 / 2009$ & $10 / 05 / 2016$ & $26 / 10 / 2017$ & $18 / 12 / 2020$ \\
\hline ARCH-LM & $462.687^{*}$ & $21.554^{* *}$ & 0.427 & $20.070^{* * *}$ & 9.216 & $38.621^{*}$ & $124.756^{*}$ \\
\hline$\alpha$ & $0.038^{*}$ & $0.135^{*}$ & -0.001 & $0.116^{*}$ & $0.047^{* *}$ & $0.098 * * *$ & $0.264^{*}$ \\
\hline$\beta$ & $0.962^{*}$ & $0.732^{*}$ & 0.996 & $0.83^{*}$ & $0.801^{*}$ & $0.811^{*}$ & 0.709 \\
\hline$\alpha+\beta$ & 1.000 & 0.867 & 0.995 & 0.946 & 0.848 & 0.909 & 0.973 \\
\hline Half-life & 7219.937 & 4.857 & 138.283 & 12.486 & 4.204 & 7.236 & 25.102 \\
\hline t-df & $3.840^{*}$ & $5.143^{*}$ & $3.872^{*}$ & $3.739^{*}$ & $3.991 *$ & $6.135^{*}$ & $5.061^{*}$ \\
\hline$\gamma \mathrm{GJR}$ & $0.036^{*}$ & $0.111^{* * *}$ & 0.001 & $0.230^{*}$ & $0.119^{*}$ & $0.203^{* *}$ & $0.150 * *$ \\
\hline$\gamma$ EGARCH & $-0.029 *$ & -0.046 & -0.002 & $-0.151^{*}$ & $-0.107^{*}$ & $-0.169^{*}$ & $-0.067 * * *$ \\
\hline BIC GARCH & 4.756 & 6.434 & 4.514 & 5.251 & 4.146 & 3.132 & 4.051 \\
\hline BIC GJR & 4.754 & 6.436 & 4.527 & 5.233 & 4.135 & 3.132 & 4.059 \\
\hline BIC EGARCH & 4.736 & 6.431 & 4.517 & 5.223 & 4.129 & 3.129 & 4.055 \\
\hline
\end{tabular}

ARCH-LM test. $*, * *, * * *$ denote statistically significant at the $1 \%, 5 \%$ and $10 \%$ significance levels, respectively. $\alpha+\beta=1$ is the measure of volatility persistence. Half-life gives the point estimate of the half-life in days given as $H L=\frac{\log (0.5)}{\log (\alpha+\beta)}$. $\mathrm{t}$-df represents the Student's $t$ degrees of freedom. GARCH, GJR and EGARCH are conditional heteroskedastic models defined in (4),

(6) and (5), respectively.

Table 15 GARCH, GJR and EGARCH(1,1), Persistence and Half-life - TESLA

\begin{tabular}{lrrrrr} 
& Full sample & $08 / 05 / 2014$ & $26 / 03 / 2018$ & $28 / 01 / 2020$ & $18 / 12 / 2020$ \\
\hline ARCH-LM & $165.073^{*}$ & $34.563^{*}$ & 16.895 & 12.521 & $23.59^{* *}$ \\
$\alpha$ & $0.041^{*}$ & $0.134^{* *}$ & 0.111 & 0.086 & $0.160^{* *}$ \\
$\beta$ & $0.948^{*}$ & $0.553^{*}$ & $0.662^{* *}$ & $0.694^{* *}$ & $0.784^{*}$ \\
$\alpha+\beta$ & $\mathbf{0 . 9 8 9}$ & $\mathbf{0 . 6 8 8}$ & $\mathbf{0 . 6 7 3}$ & $\mathbf{0 . 7 8 0}$ & $\mathbf{0 . 9 4 5}$ \\
Half-life & 60.701 & 1.850 & 1.753 & 2.786 & 12.145 \\
t-df & $3.542^{*}$ & $3.802^{*}$ & $4.475^{*}$ & $3.367^{*}$ & $5.908^{* * *}$ \\
$\gamma$ GJR & -0.004 & -0.010 & $0.127^{*}$ & 0.186 & 0.005 \\
$\gamma$ EGARCH & -0.008 & 0.008 & $-0.189^{*}$ & -0.088 & -0.038 \\
BIC GARCH & $\mathbf{5 . 0 7 6}$ & $\mathbf{5 . 2 9 4}$ & 4.571 & $\mathbf{5 . 2 5 0}$ & $\mathbf{6 . 3 7 3}$ \\
BIC GJR & 5.080 & 5.300 & $\mathbf{4 . 5 6 3}$ & 5.259 & 6.3967 \\
BIC EGARCH & 5.077 & 5.297 & 4.571 & 5.257 & 6.393 \\
\hline
\end{tabular}

ARCH-LM test. $*, * *, * * *$ denote statistically significant at the $1 \%, 5 \%$ and $10 \%$ significance levels, respectively. $\alpha+\beta=1$ is the measure of volatility persistence. Half-life gives the point estimate of the half-life in days given as $H L=\frac{\log (0.5)}{\log (\alpha+\beta)} . t-d f$ represents the Student's $t$ degrees of freedom. GARCH, GJR and EGARCH are conditional heteroskedastic models defined in (4),

(6) and (5), respectively.

estimates, as pointed by [40], sometimes masks important differences in persistence across sub-samples. If we consider all the sub-samples, the estimates range between 0.207 (Facebook) and almost 1.000 (NASDAQ).

Table 19 shows the estimates for the two most recent sub-samples. As one can see, the persistence is always higher among the US stock indices, taking longer to cancel the effects of shocks on volatility. Due to the COVID-19, the persistence has increased in 2020, but the estimates remain far from 1 in case of FATANG stocks. If we go back to the period before COVID-19, only Google has a similar behavior when compared to the US indices. Thus, we can conclude that bad news about FATANG stocks seem to have a shorter impact on volatility. 
Table 16 GARCH, GJR and EGARCH(1,1), Persistence and Half-life - APPLE

\begin{tabular}{lrrrrrr} 
& Full sample & $30 / 06 / 1997$ & $03 / 10 / 2000$ & $26 / 05 / 2009$ & $20 / 02 / 2020$ & $18 / 12 / 2020$ \\
\hline ARCH-LM & $42.814^{*}$ & $158.408^{*}$ & 9.040 & $97.877^{*}$ & $60.108^{*}$ & $39.313^{*}$ \\
$\alpha$ & $0.061^{*}$ & $0.036^{*}$ & $0.198^{*}$ & $0.029^{*}$ & $0.101^{*}$ & $0.139^{* *}$ \\
$\beta$ & $0.938^{*}$ & $0.893^{*}$ & $0.543^{*}$ & $0.961^{*}$ & $0.846^{*}$ & $0.834^{*}$ \\
$\alpha+\beta$ & $\mathbf{0 . 9 9 9}$ & $\mathbf{0 . 9 2 9}$ & $\mathbf{0 . 7 4 1}$ & $\mathbf{0 . 9 9 0}$ & $\mathbf{0 . 9 4 7}$ & $\mathbf{0 . 9 7 3}$ \\
Half-life & 519.644 & 9.412 & 2.312 & 68.968 & 12.761 & 25.652 \\
t-df & $4.597^{*}$ & $4.041^{*}$ & 5.256 & $5.801^{*}$ & $4.463^{*}$ & $4.525^{*}$ \\
$\gamma$ GJR & $0.039^{*}$ & $0.083^{*}$ & 0.015 & $0.040^{*}$ & $0.208^{*}$ & 0.162 \\
$\gamma$ EGARCH & $-0.033^{*}$ & $-0.061^{*}$ & 0.015 & $-0.045^{*}$ & $-0.138^{*}$ & -0.115 \\
BIC GARCH & 4.533 & 4.809 & $\mathbf{5 . 6 2 0}$ & 4.867 & 3.656 & $\mathbf{4 . 9 4 7}$ \\
BIC GJR & 4.533 & 4.807 & 5.628 & 4.866 & 3.636 & 4.960 \\
BIC EGARCH & $\mathbf{4 . 5 2 1}$ & $\mathbf{4 . 8 0 5}$ & 5.634 & $\mathbf{4 . 8 6 4}$ & $\mathbf{3 . 6 2 8}$ & 4.962 \\
\hline
\end{tabular}

ARCH-LM test. *, **, *** denote statistically significant at the $1 \%, 5 \%$ and $10 \%$ significance levels, respectively. $\alpha+\beta=1$ is the measure of volatility persistence. Half-life gives the point estimate of the half-life in days given as $H L=\frac{\log (0.5)}{\log (\alpha+\beta)} \cdot \mathrm{t}$-df represents

the Student's $t$ degrees of freedom. GARCH, GJR and EGARCH are conditional heteroskedastic models defined in (4), (6) and (5), respectively.

Table 17 GARCH, GJR and EGARCH(1,1), Persistence and Half-life - NETFLIX

\begin{tabular}{lrrrrr} 
& Full sample & $18 / 10 / 2004$ & $14 / 09 / 2011$ & $23 / 04 / 2013$ & $18 / 12 / 2020$ \\
\hline ARCH-LM & $32.096^{*}$ & 11.149 & 8.961 & 1.430 & 9.864 \\
$\alpha$ & $0.031^{*}$ & $0.235^{* *}$ & $0.089^{*}$ & 0.177 & $0.110^{*}$ \\
$\beta$ & $0.963^{*}$ & $0.377^{* * *}$ & $0.761^{*}$ & 0.251 & $0.822^{*}$ \\
$\alpha+\beta$ & $\mathbf{0 . 9 9 4}$ & $\mathbf{0 . 6 1 2}$ & $\mathbf{0 . 8 5 0}$ & $\mathbf{0 . 4 2 8}$ & $\mathbf{0 . 9 3 1}$ \\
Half-life & 109.711 & 1.412 & 4.265 & 0.817 & 9.729 \\
t-df & $3.175^{*}$ & $3.217^{*}$ & $3.526^{*}$ & $2.710^{*}$ & $3.357^{*}$ \\
$\gamma$ GJR & $0.018^{* *}$ & $0.396^{* * *}$ & 0.051 & -0.131 & $0.153^{*}$ \\
$\gamma$ EGARCH & $-0.029^{*}$ & $-0.169^{* *}$ & -0.021 & 0.032 & $-0.072^{*}$ \\
SIC GARCH & 4.995 & $\mathbf{5 . 9 7 5}$ & 5.011 & $\mathbf{5 . 6 3 5}$ & 4.570 \\
SIC GJR & 4.996 & 5.979 & 5.014 & 5.643 & 4.564 \\
SIC EGARCH & $\mathbf{4 . 9 7 5}$ & 5.985 & $\mathbf{5 . 0 0 7}$ & 5.638 & $\mathbf{4 . 5 4 9}$ \\
\hline
\end{tabular}

ARCH-LM test. $*, * *, * *$ denote statistically significant at the $1 \%, 5 \%$ and $10 \%$ significance levels, respectively. $\alpha+\beta=1$ is the measure of volatility persistence. Half-life gives the point estimate of the half-life in days given as $H L=\frac{\log (0.5)}{\log (\alpha+\beta)} \cdot \mathrm{t}-\mathrm{df}$ represents the Student's $t$ degrees of freedom. GARCH, GJR and EGARCH are conditional heteroskedastic models defined in (4),

(6) and (5), respectively.

Persistence for whole the sample is also substantially higher when compared to the persistence for each sub-sample; thus, splitting the sample according to the structural breaks can reduce the overall persistence. For example, in case of Facebook, the estimated half-life ${ }^{6}$ of the volatility persistence decreases from 157 days (when the whole sample is considered) to approximately to 2 and 5 days in the most recent sub-samples, which implies that a shock is expected to lose half of its original impact in just two or five days after the structural breaks are considered (see Table 13). According to this stylized fact of the returns' volatility, FATANG stocks are also substantially different

\footnotetext{
${ }^{6}$ Half-life gives the point estimate of the hal-life in days given as $H L=\frac{\log (0.5)}{\log (\alpha+\beta)}$.
} 
Table 18 GARCH, GJR and EGARCH(1,1), Persistence and Half-life - GOOGLE

\begin{tabular}{lrrrrrr} 
& Full sample & $12 / 05 / 2006$ & $07 / 11 / 2007$ & $23 / 01 / 2009$ & $20 / 02 / 2020$ & $18 / 12 / 2020$ \\
\hline ARCH-LM & $66.915^{*}$ & $27.295^{*}$ & 5.704 & 9.410 & 17.472 & $34.527^{*}$ \\
$\alpha$ & $0.063^{*}$ & $0.090^{* *}$ & 0.036 & 0.081 & $0.030^{*}$ & $0.161^{* *}$ \\
$\beta$ & $0.927^{*}$ & $0.838^{*}$ & 0.471 & $0.873^{*}$ & $0.954^{*}$ & $0.797^{*}$ \\
$\alpha+\beta$ & $\mathbf{0 . 9 9 0}$ & $\mathbf{0 . 9 2 8}$ & $\mathbf{0 . 5 0 7}$ & $\mathbf{0 . 9 5 4}$ & $\mathbf{0 . 9 8 4}$ & $\mathbf{0 . 9 5 9}$ \\
Half-life & 66.308 & 9.276 & 1.02 & 14.719 & 44.042 & 16.422 \\
t-df & $3.889^{*}$ & $4.469^{*}$ & $5.471^{*}$ & $4.149^{*}$ & $3.834^{*}$ & $4.872^{* *}$ \\
$\gamma$ GJR & $0.071^{*}$ & 0.032 & -0.046 & $0.156^{* * *}$ & $0.058^{*}$ & $0.258^{* *}$ \\
$\gamma$ EGARCH & $-0.052^{*}$ & -0.019 & -0.016 & $-0.254^{*}$ & $-0.065^{*}$ & $-0.120^{* * *}$ \\
BIC GARCH & 3.756 & $\mathbf{4 . 5 9 3}$ & $\mathbf{3 . 6 6 7}$ & 5.183 & 3.459 & $\mathbf{4 . 5 7 7}$ \\
BIC GJR & 3.751 & 4.607 & 3.682 & $\mathbf{5 . 1 5 8}$ & 3.453 & 4.579 \\
BIC EGARCH & $\mathbf{3 . 7 4 2}$ & 4.611 & 3.685 & 5.204 & $\mathbf{3 . 4 4 4}$ & 4.585 \\
\hline
\end{tabular}

ARCH-LM test. $*, * *, * * *$ denote statistically significant at the $1 \%, 5 \%$ and $10 \%$ significance levels, respectively. $\alpha+\beta=1$ is the measure of volatility persistence. Half-life gives the point estimate of the half-life in days given as $H L=\frac{\log (0.5)}{\log (\alpha+\beta)}$. $\mathrm{t}$-df represents the Student's $t$ degrees of freedom. GARCH, GJR and EGARCH are conditional heteroskedastic models defined in (4),

(6) and (5), respectively.

Table 19 Volatility persistence in the sub-samples before and after COVID-19

\begin{tabular}{lrrrr} 
& \multicolumn{2}{c}{ Before COVID-19 } & \multicolumn{2}{c}{ After COVID-19 } \\
Indices/Stocks & $\alpha+\beta$ & Half-life & $\alpha+\beta$ & Half-life \\
\hline S\&P 500 & 0.965 & 19.654 & $\mathbf{0 . 9 9 1}$ & 76.704 \\
DJIA & 0.984 & 42.418 & $\mathbf{0 . 9 9 7}$ & 272.224 \\
NASDAQ & 0.977 & 29.802 & $\mathbf{1 . 0 0 0}$ & 1571.416 \\
FACEBOOK & 0.736 & 2.259 & 0.867 & 4.853 \\
AMAZON & 0.909 & 7.236 & 0.973 & 25.102 \\
TESLA & $\mathbf{0 . 7 8 0}$ & 2.786 & 0.945 & 12.145 \\
APPLE & 0.947 & 12.761 & 0.973 & 25.652 \\
NETFLIX & $\mathbf{0 . 4 2 8}$ & 0.817 & 0.931 & 9.729 \\
GOOGLE & 0.984 & 44.042 & 0.959 & 16.422 \\
\hline
\end{tabular}

from the US indices. After COVID-19 the estimated half-life ranges between 4.853 (Facebook) and 25.652 (Apple), while the estimate for US indices ranges between 76.704 (S\&P 500) and 1571.224 (NASDAQ, where the GARCH(1,1) process is highly persistent, almost-integrated).

Finally, what to conclude about the asymmetric effect on volatility? Despite taking into consideration the financial asset and the sub-sample, the asymmetric GJR and EGARCH models almost completely dominate the symmetric GARCH model, which means that negative shocks (when compared to the positive ones) have a stronger impact on returns volatility. The estimates for the asymmetric coefficient $\gamma$ have the correct sign (positive in the case of GJR and negative in the case of EGARCH), and they are statistically significant for most of the sub-samples. The Bayesian Information Criterion (BIC) also favours the asymmetric conditional heteroskedasticity models. There are just a few exceptions where positive and negative shocks have the same impact on volatility and the symmetric GARCH beats the other two models. In the most recent sub-sample, after COVDI-19, the estimate for the coefficient of asymmetry $(\gamma)$ in not statistically significant in cases of the three US indices: 
S\&P 500, NASDAQ (EGARCH estimate), DJIA plus Tesla and Apple. Thus, it seems that bad news still have higher (even shorter) impact on volatility in case of FATANG stocks; the difference between the impact of positive and negative news on volatility seems lower in case of US indices.

\section{Conclusion}

Volatility of financial asset returns has been extensively studied for the last thirty years and it remains a very important topic of investigation due to its importance for investors, financial analysts and academics. In this paper, we analyze how the volatility of returns of three US stock indices: S\&P500, DJIA and NASDAQ, and six American stocks under the acronym FATANG: Facebook, Amazon, Tesla, Apple, Netflix and Google, representing the new economy, have evolved in recent decades.

First we analyze dispersion, skewness and kurtosis of the empirical distributions of returns. To notice the temporal changes, all the measures are computed over a rolling window encompassing the previous year of daily observations $(\mathrm{T}=250)$. Based on the unconditional standard deviation we observe a sharp decrease in volatility after the year 2012 regardless of the index or the stock being considered. For Amazon and Apple, for example, the variance of returns before 2012 was almost 6 and 4 times higher than the variance computed for the next six years. In terms of skewness and kurtosis, when the stock indices are considered, most of the estimates of skewness are negative and just a few are statistically significant and positive. Furthermore, the peaks in the kurtosis are mostly due to large negative returns, giving rise to the negative spikes of the coefficient of skewness. The conclusions are different when the individual FATANG stocks are analyzed. The estimates of skewness can be either positive or negative and the peaks of kurtosis are also explained by large positive returns leading to the positive spikes of the coefficient of skewness. Thus, large positive returns also inflate the kurtosis. By separating the "bad" from "good" volatility and kurtosis, because the former represents risk while the latter only represents uncertainty, and based on semi-variance and semikurtosis measures, we conclude that while volatility and kurtosis of FATANG stocks returns represent more uncertainty than risk, they represent more risk than uncertainty in the case of stock indices. Thus, the indices seem to be riskier than that particular class of tech stocks.

Second, we analyze the autocorrelation of returns, absolute returns and squared returns. According to the Ljung-Box test for returns, the autocorrelation seems not to be relevant (only in a small percentage of windows is the white noise null hypothesis rejected, with the exception being the NASDAQ composite index with more than $20 \%$ of rejections). The autocorrelation structure of returns still seems weaker in the case of individual stocks (in case of Tesla, for example, the number of rejections is almost zero). Even though the series of returns seem to be weakly correlated over time, the autocorrelation of absolute and squared returns is stronger, pointing to a positive autocorrelation 
over several days, which quantifies the fact that high volatility events tend to cluster in time. However, the volatility clustering stylized fact is not so evident for individual stocks, especially in cases of Amazon, Tesla and Netflix. The empirical results seem also to confirm that sample autocorrelations for absolute returns are greater than the sample autocorrelations for squared returns. As one possible explanation for the large positive autocorrelation between $\left|r_{t}\right|$ and $r_{t}^{2}$ is the heteroskedasticity of returns, i.e., the variance or conditional variance is changing over time, we computed also the ARCH-LM test. The results suggest that all financial asset returns exhibit ARCH effects and conditional heteroskedasticity. However, this empirical result is not so evident in the case of FATANG stocks.

Finally, we model time-varying conditional volatility. As several studies show that structural breaks have potential implications regarding the estimation results, we first tested for the existence of structural breaks in volatility of the twelve returns series by applying a modified version of the iterated cumulative sum of squares (ICSS) algorithm that allows for temporal dependence. The results show strong evidence of structural breaks in the unconditional variance for all the returns series, leading to distinct regimes in volatility. Next we estimated an $\operatorname{ARMA}(4,0)$ model for the conditional mean in combination with $\operatorname{GARCH}(1,1), \operatorname{GJR}(1,1)$ and $\operatorname{EGARCH}(1,1)$ models for the conditional variance across the sub-samples resulting from the structural breaks identified by the modified ICSS algorithm.

Three main conclusions can be drawn. First, the ARCH-LM test points for the existence of conditional heteroskedasticity in most of the series and for most of the sub-samples resulting from the structural breaks. However, in the most recent past there are four exceptions: Facebook, Tesla, Netlix and Google. Thus, the suspicion of constant variance of returns is more evident among the FATANG stocks. Second, GARCH $(1,1)$ processes are highly persistent (almost-integrated) when estimated over the full sample, with the estimate for $\alpha+\beta$ ranging between 0.989 and 1. If we focus mainly on the sub-sample resulting from the last structural break, the estimates range between 0.867 (Facebook) and 1 (NASDAQ). The decrease in volatility persistence is also a symptom of less volatility in the stock markets. Finally, the asymmetric GJR and EGARCH models almost completely dominate, despite taking into consideration the financial asset and the sub-sample being considered, the symmetric GARCH model, which means that negative shocks (when compared to the positive ones) have a stronger impact on returns volatility.

In conclusion, major empirical findings point to volatility decreasing in the most recent past, excluding March 2020. Thus, should we keep faith with homoskedasticity or trust that a new heteroskedasticity regime will come soon? Is the 2020 increase in volatility evidence of on-going structural change, or is just a scare for investors? Answers are required for a new million dollar question. 


\section{Conflict of interest}

The authors declare that they have no conflict of interest.

\section{References}

1. Anufriev, M., Gardini, L. and Radi, D. (2020) Chaos, border collisions and stylized empirical facts in an asset pricing model with heterogeneous agents. Nonlinear Dyn 102, 993-1017.

2. Bai, L., Wei, Y., Wei, G., Li, X. and Zhang, S. (2020) Infectious disease pandemic and permanent volatility of international stock markets: A long-term perspective, Finance Research Letters, in Press.

3. Black, F. (1976) Studies of stock price volatility changes, Proceedings of the 1976 Meeting of the Business and Economic Statistics Section, 177-181.

4. Bollerslev, T. (1986) Generalized autoregressive conditional heteroskedasticity, Journal of Econometrics, 31 (3), 307-327.

5. Bollerslev, T. (1987) A Conditionally Heteroskedastic Time Series Model for Speculative Prices and Rates of Return, The Review of Economics and Statistics, 69 (3), $542-547$.

6. Bollerslev, T., Chou, R. and Kroner, K. (1992) Arch modelling in finance, Journal of Econometrics, 52, 5-59.

7. Cont, R. (2001) Empirical properties of asset returns: stylized facts and statistical issues, Quantitative Finance, 1 (2), 223-236.

8. Curto, J. D. and Tomaz, J. and Pinto, J. C. (2009) A new approach to bad news effects on volatility: the multiple-sign-volume sensitive regime EGARCH model (MSVEGARCH), Portuguese Economic Journal, 8 (1), 23-36.

9. Curto, J.D. and Pinto, J.C. and Tavares, G. (2009) Modeling stock markets' volatility using GARCH models with Normal, Student's $t$ and stable Paretian distributions, Statistical Papers, 50 (2), 311-321.

10. Curto, J.D. and Pinto, J.C. (2012) Predicting the Financial Crisis Volatility, Economic Computation And Economic Cybernetics Studies and Research Journal, 46 (1), 183195.

11. De Pooter, M. and van Dijk, D. (2004), Testing for changes in volatility in heteroskedastic time series - a further examination, Erasmus University Rotterdam, Erasmus School of Economics (ESE), Econometric Institute, Econometric Institute Research Papers, EI 2004-38.

12. Ding, Z. and Granger, C. and Engle, R. (1993) A long memory property of stock market returns and a new model, Journal of Empirical Finance, 1 (1), 83-106.

13. Engle, R. (1982) Autoregressive Conditional Heteroscedasticity with Estimates of the Variance of United Kingdom Inflation, Econometrica, 50 (4), 987-1007.

14. Engle, R. and Bollerslev, T. (1986) Modelling the persistence of conditional variances, Econometric Reviews, 5 (1), 1-50.

15. Fama, E. (1965) The Behaviour of Stock Market Prices, The Journal of Business, 64, 34-105.

16. Fama, E. (1970) Efficient Capital Markets: A Review of Theory and Empirical Work, Journal of Finance, 25(2), 383-417.

17. Glosten, L., Jagannanthan, R. and Runkle, R. (1993), it On the relationship between the expected value and the volatility of the nominal excess returns on stocks, Journal of Finance, 48, 1779-1801.

18. Granger, C.W.J. and Ding, Z. (1995) Some Properties of Absolute Return: An Alternative Measure of Risk, Annals of Economics and Statistics, 40, 67-91.

19. Granger, C.W.J. (2005) The Present and Future of Empirical Finance, Financial Analysts Journal, 61 (4), 15-18.

20. Hansen, P. and Lunde, A. (2005), A forecast comparison of volatility models: does anything beat a $\operatorname{GARCH}(1,1)$ ?, Journal of Applied Econometrics, 20, 873-889. 
21. Hillebrand, E. (2004) Neglecting parameter changes in GARCH models, Journal of Econometrics, 129 (1-2), 121-138.

22. Hsieh, D. A. (1989) Testing for Nonlinear Dependence in Daily Foreign Exchange Rates, The Journal of Business, 62 (3), 339-68.

23. Inclán, C. and G. Tiao (1994) Use of Cumulative Sums of Squares for Retrospective Detection of Changes in Variance, Journal of American Statistical Association, 89, 913-923.

24. Lamoureux, C. G. and Lastrapes, W. D. (1990) Persistence in Variance, Structural Change, and the GARCH Model, Journal of Business \& Economic Statistics, 8 (2), $225-234$.

25. Killick, R. and Fearnhead, P. and Eckley, I.A. (2012) Optimal Detection of Changepoints With a Linear Computational Cost, Journal of the American Statistical Association, 107, 1590-1598.

26. Kim, s. and Cho, S. and Lee, S. (2000) On the Cusum test for parameter changes in garch(1,1) Models, Communications in Statistics - Theory and Methods, 29 (2), 445-462.

27. Kokoszka, P. and Leipus, R. (2000) Change-point estimation in ARCH models, Bernoulli, 6 (3), 513-539.

28. Liu, S.-M. and Brorsen, B. (1995) Maximum Likelihood Estimation of a Garch-Stable Model, Journal of Applied Econometrics, 10 (3), 273-85.

29. Mandelbrot, B. (1963) The Variation of Certain Speculative Prices, The Journal of Business, 36.

30. Maris, K., Pantou, G., Nikolopoulos, K., Pagourtzi, E. and Assimakopoulos, V. (2004), A Study of Financial Volatility Forecasting Techniques in the FTSE/ASE 20 Index, Applied Economics Letters, 11, 453-457.

31. Markowitz, H. M. (1959), Portfolio Selection: Efficient Diversification of Investments, John Wiley \& Sons, New York.

32. Menezes, R., Ferreira, N. B. and Mendes, D. (2006) Co-Movements and Asymmetric Volatility in the Portuguese and U.S. Stock Markets. Nonlinear Dyn 44, 359-366.

33. Mikosch, T. and Stărică, C. (2003) Long Range Dependence and ARCH Modeling, in Theory and Applications of Long Range Dependence, Ed. Doukham, P. and Oppenheim, G. and Taqqu. M., 439-460, Birkhäuser, Boston.

34. Mikosch, T. and Stărică, C. (2004) Nonstationarities in Financial Time Series, the Long-Range Dependence, and the IGARCH Effects, The Review of Economics and Statistics, 86 (1), 378-390.

35. Mittnik, S. and Paolella, M. and Rachev, S. (1998) Unconditional and Conditional Distributional Models for the Nikkei Index, Asia-Pacific Financial Markets, 5(2), 99128.

36. Morales, L. and Gassie, E. (2011) Structural breaks and financial volatility: Lessons from BRIC countries, Leibniz Institute of Agricultural Development in Central and Eastern Europe (IAMO), 13.

37. Nelson D. B. (1991), Conditional heteroskedasticity in asset returns: A new approach, Econometrica, 59, 347-370.

38. Perron, P. and Qu, Z. (2006), An Analytical Evaluation of the Log-periodogram Estimate in the Presence of Level Shifts and its Implications for Stock Returns Volatility, Boston University - Department of Economics - Working Papers Series, WP2006-016.

39. Poterba, J. and Summers, L. (1986), The Persistence of Volatility and Stock Market Fluctuations, American Economic Review, 76, 1142-1151.

40. Rapach, D. E. and Strauss, J. K. (2008) Structural breaks and GARCH models of exchange rate volatility, Journal of Applied Econometrics, 23 (1), 65-90.

41. Ribeiro, P. P. and Cermeño, R. and Curto, J. D. (2017) Sovereign bond markets and financial volatility dynamics: Panel-GARCH evidence for six euro area countries, Finance Research Letters, 21 (C), 107-114.

42. Ribeiro, P. P. and Curto, J. D. (2017) Volatility spillover effects in interbank money markets, Review of World Economics (Weltwirtschaftliches Archiv), 153 (1), 105-136.

43. Sadefo, J. K., Tassak, C. D. and Fono, L. (2011), Kurtosis and semi-kurtosis for portfolio selection with fuzzy returns. 
44. Salisu, A. A. and Vo, X. V. (2020) Predicting stock returns in the presence of COVID19 pandemic: The role of health news, International Review of Financial Analysis, 71.

45. Sanso, A. and Arago, V. and Carrion-i-Silvestre, J. (2004) Testing for changes in the unconditional variance of financial time series, Spanish Review of Financial Economics, 4, 32-53.

46. Shehzad, K., Xiaoxing, L. and Kazouz, H. (2020) COVID-19's disasters are perilous than Global Financial Crisis: A rumor or fact?, Finance Research Letters, Volume 36.

47. Shen, D., Urquhart, A. and Wang, P. (2020) Forecasting the volatility of Bitcoin: The importance of jumps and structural breaks, European Financial Management, 26 (5), 1294-1323.

48. Schwarz, G. (1978), Estimating the dimension of a model, Annals of Statistics, 6, 461-64.

49. Smith, D. (2008), Testing for structural breaks in GARCH models, Applied Financial Economics, 18 (10), 845-862.

50. Tavares, A. and Curto, J.D. and Tavares, G. (2007) Modelling Heavy Tails and Asymmetry Using ARCH-type Models With Stable Paretian Distributions, Nonlinear Dyn, 51 (1-2), 231-243.

51. Taylor, S. (1986), Modelling Financial Time Series, John Wiley \& Sons, New York.

52. Xue, W-J. (2020) Financial sector development and growth volatility: An international study, International Review of Economics \& Finance, 70, 67-88.

53. Wen, F., Xiao, J., Chuangxia, H. and Xia, X. (2018) Interaction between oil and US dollar exchange rate: nonlinear causality, time-varying influence and structural breaks in volatility, Applied Economics, 50 (3), 319-334.

54. Wooldridge, J. M. (1990) A Unified Approach to Robust, Regression-Based Specification Tests, Econometric Theory, 6, 17-43. 
Fig. 4 Rolling window mean (AA) and standard deviation (SD) - US Indices

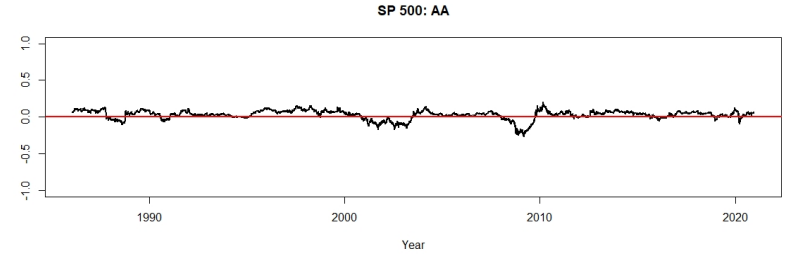

SP 500: SD
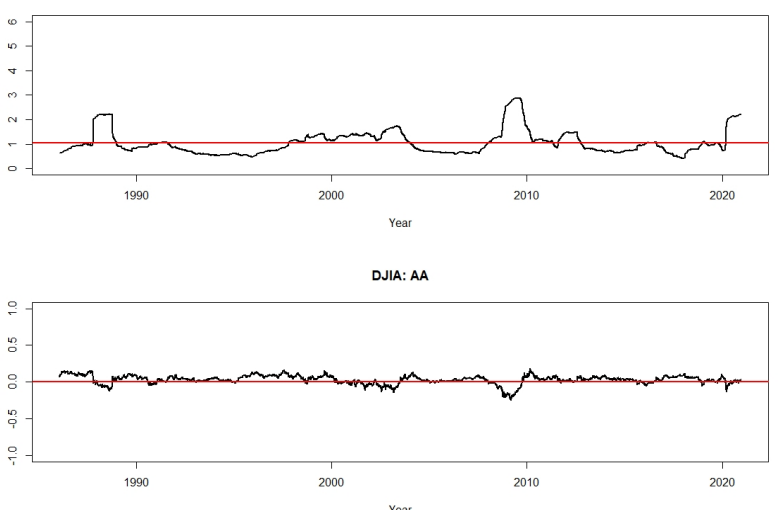

DJIA: SD

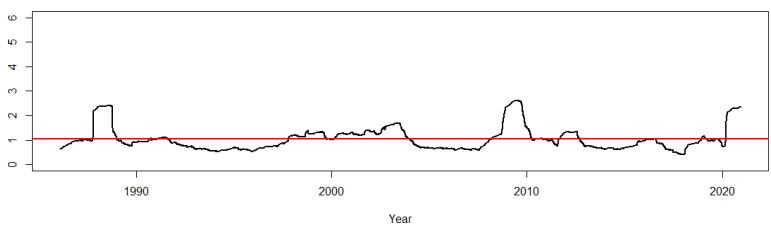

NASDAQ: AA

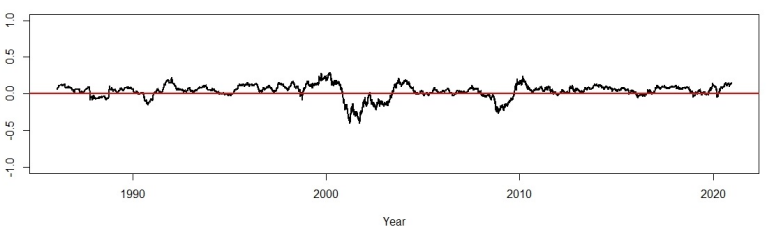

NASDAO:SD

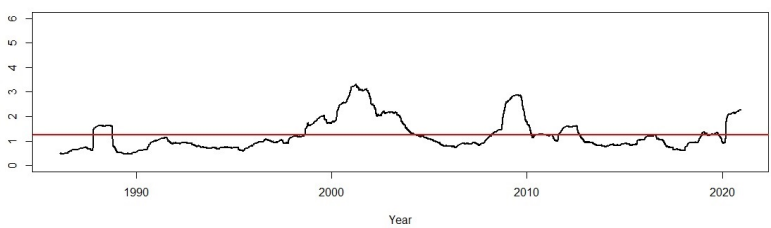

The Arithmetic Average (AA) and the Standard Deviation (SD) are computed over a rolling window encompassing the previous year of daily observations $(T=250)$. The horizontal lines represent the mean of each series. 
Fig. 5 Rolling window mean (AA) and standard deviation (SD) - FATANG stocks
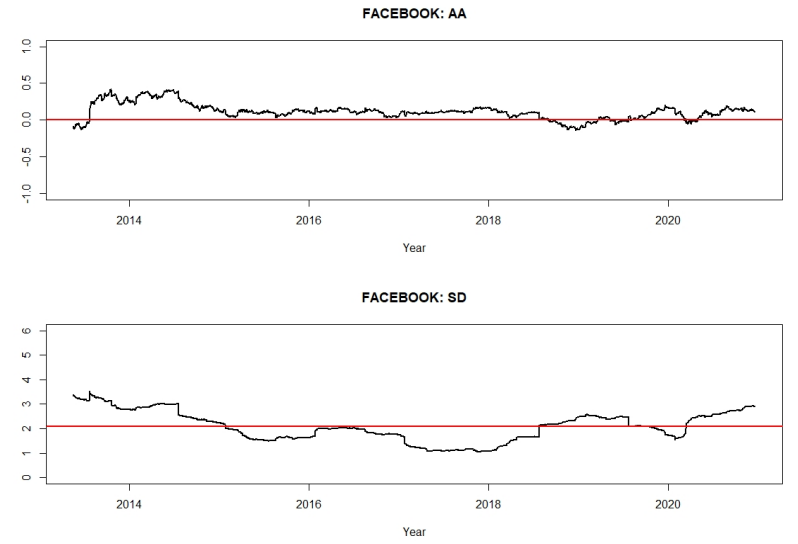

AMAZON: AA

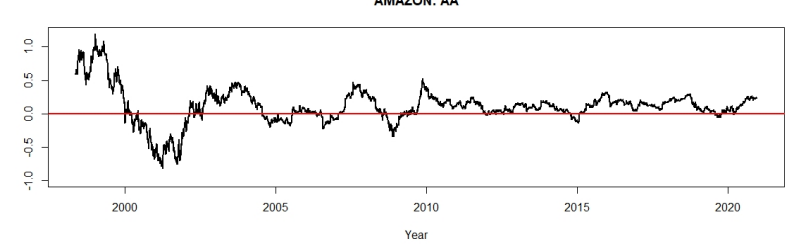

AMAZON: SD

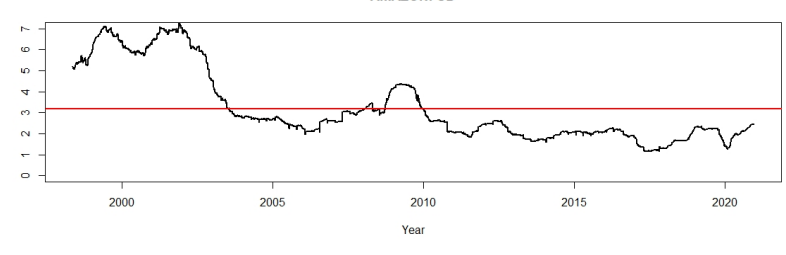

TESLA: AA

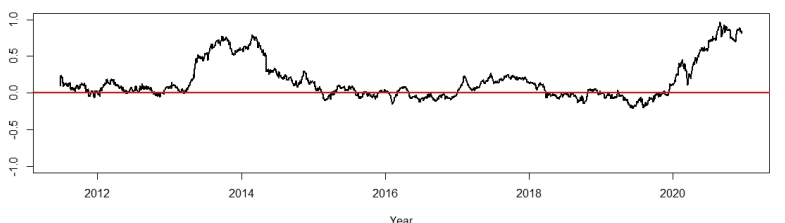

TESLA:SD

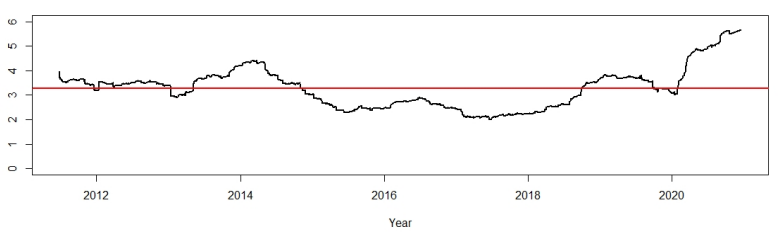

APPLE: AA
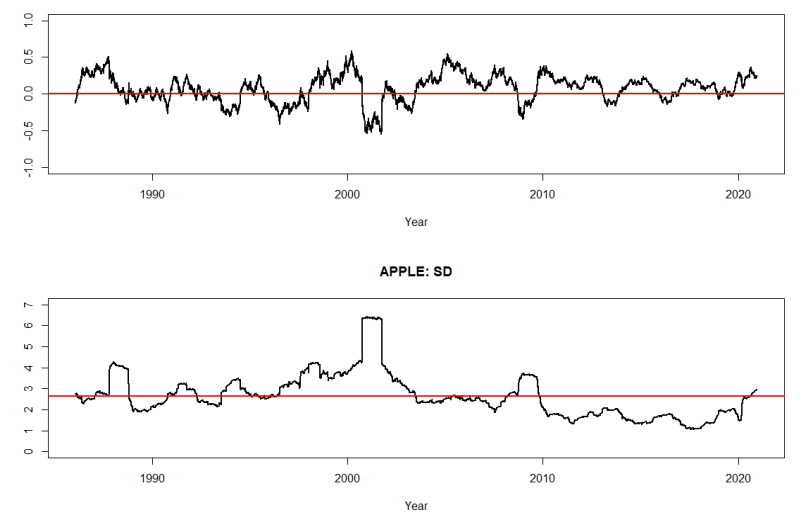

NETFLIX: AA

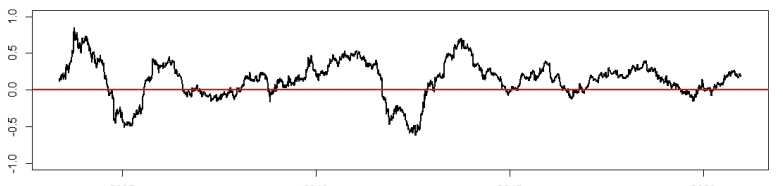


Fig. 6 Skewness and kurtosis - US indices
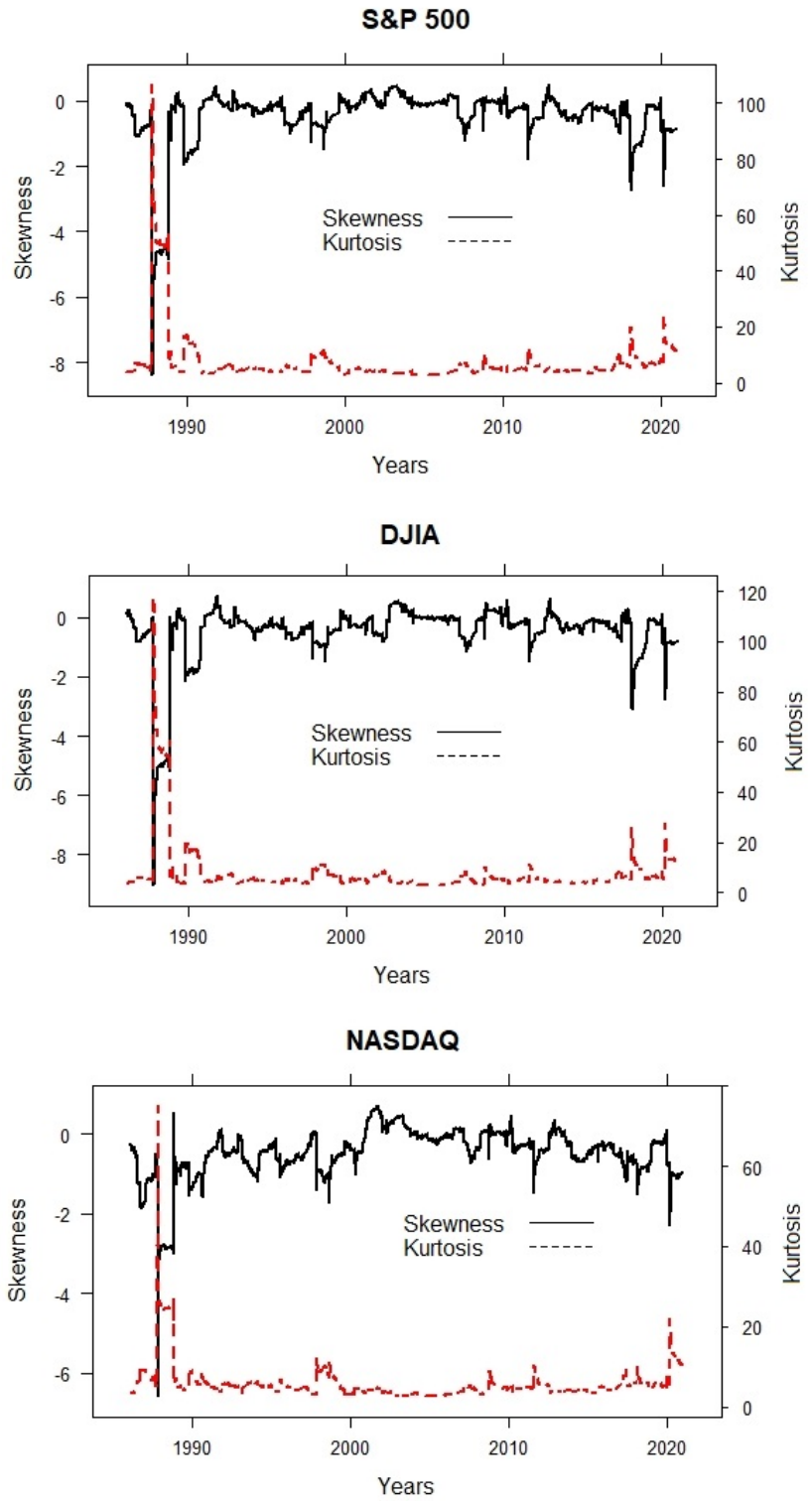
Fig. 7 Skewness and kurtosis - FATANG stocks
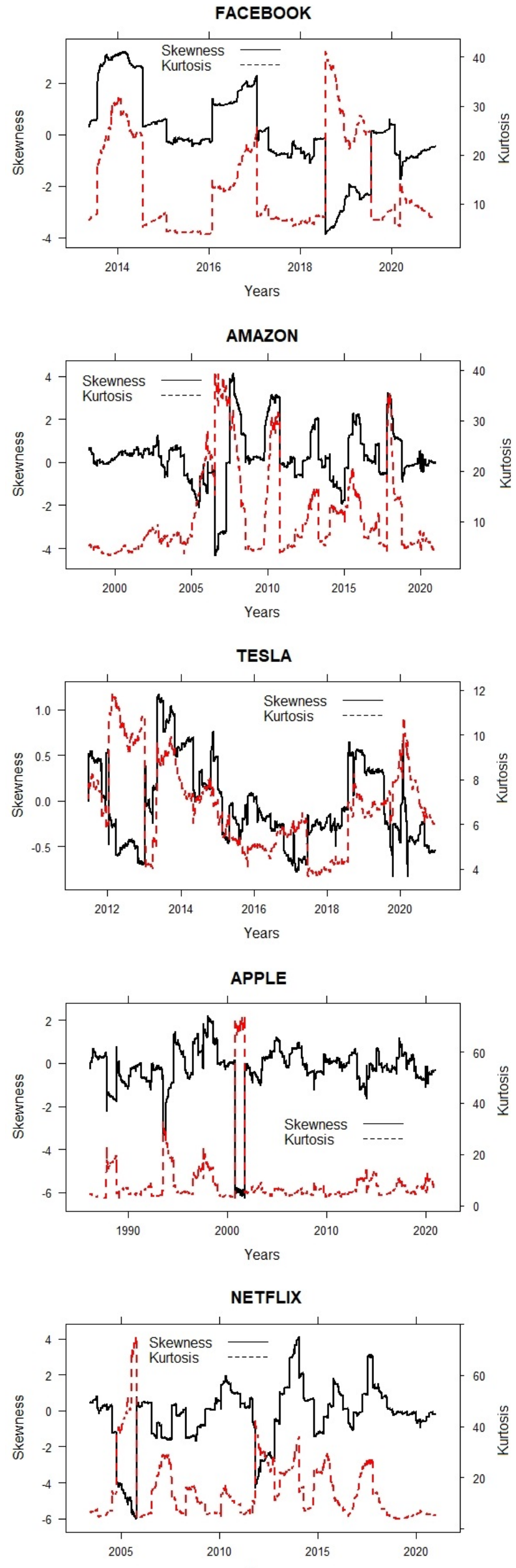
Fig. 8 Ratio of the semi-kurtosis in the kurtosis - US Indices

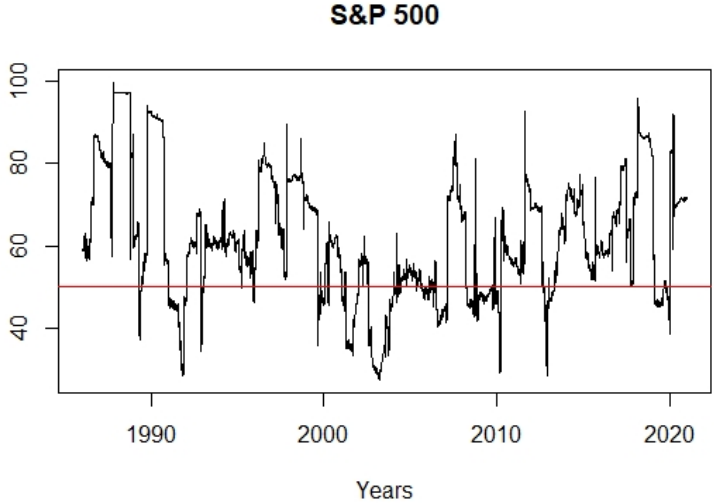

DJIA

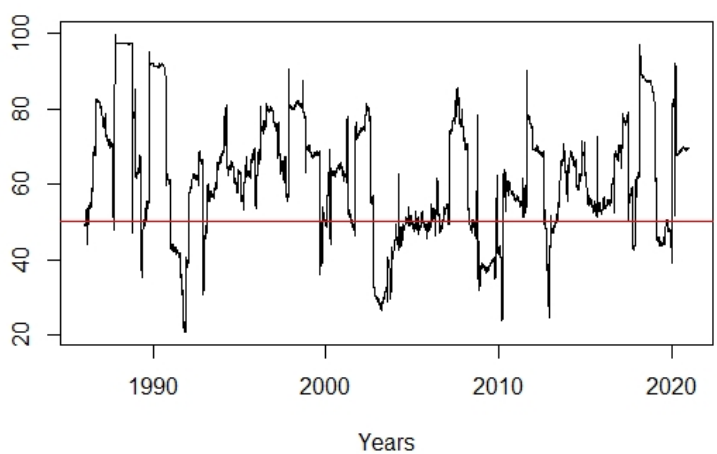

NASDAQ

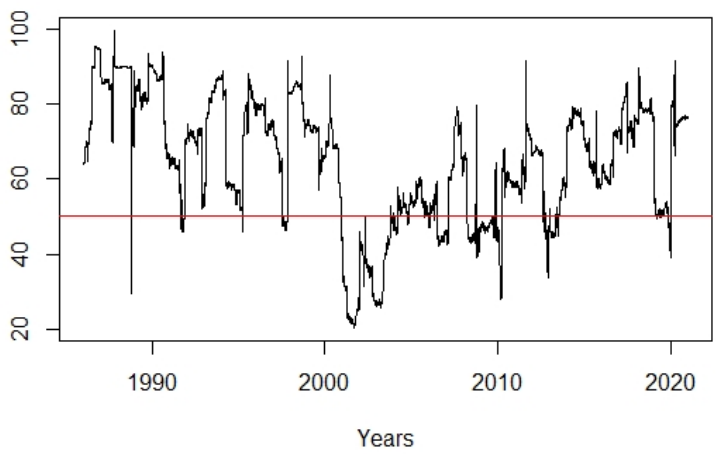


Fig. 9 Ratio of the semi-kurtosis in the kurtosis - FATANG stocks

FACEBOOK

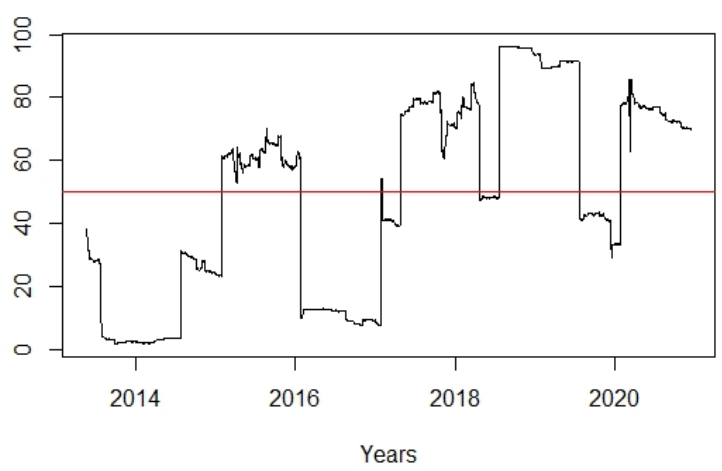

AMAZON

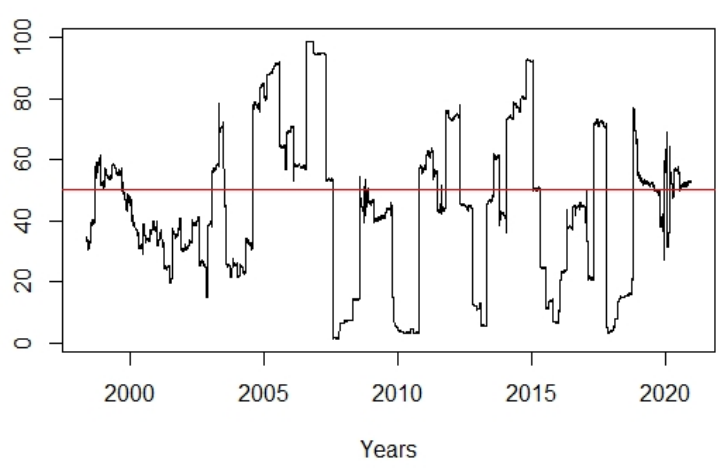

TESLA

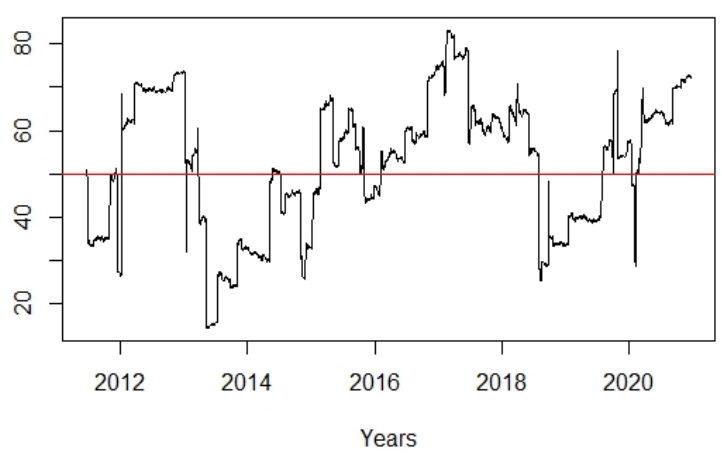

APPLE

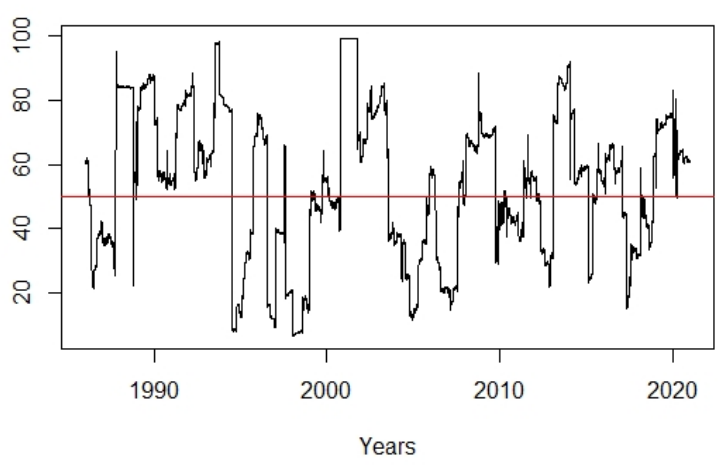

NETFLIX

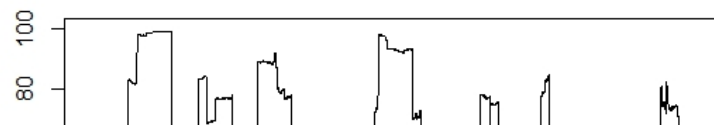


Fig. 10 Ljung-Box statistic - US indices
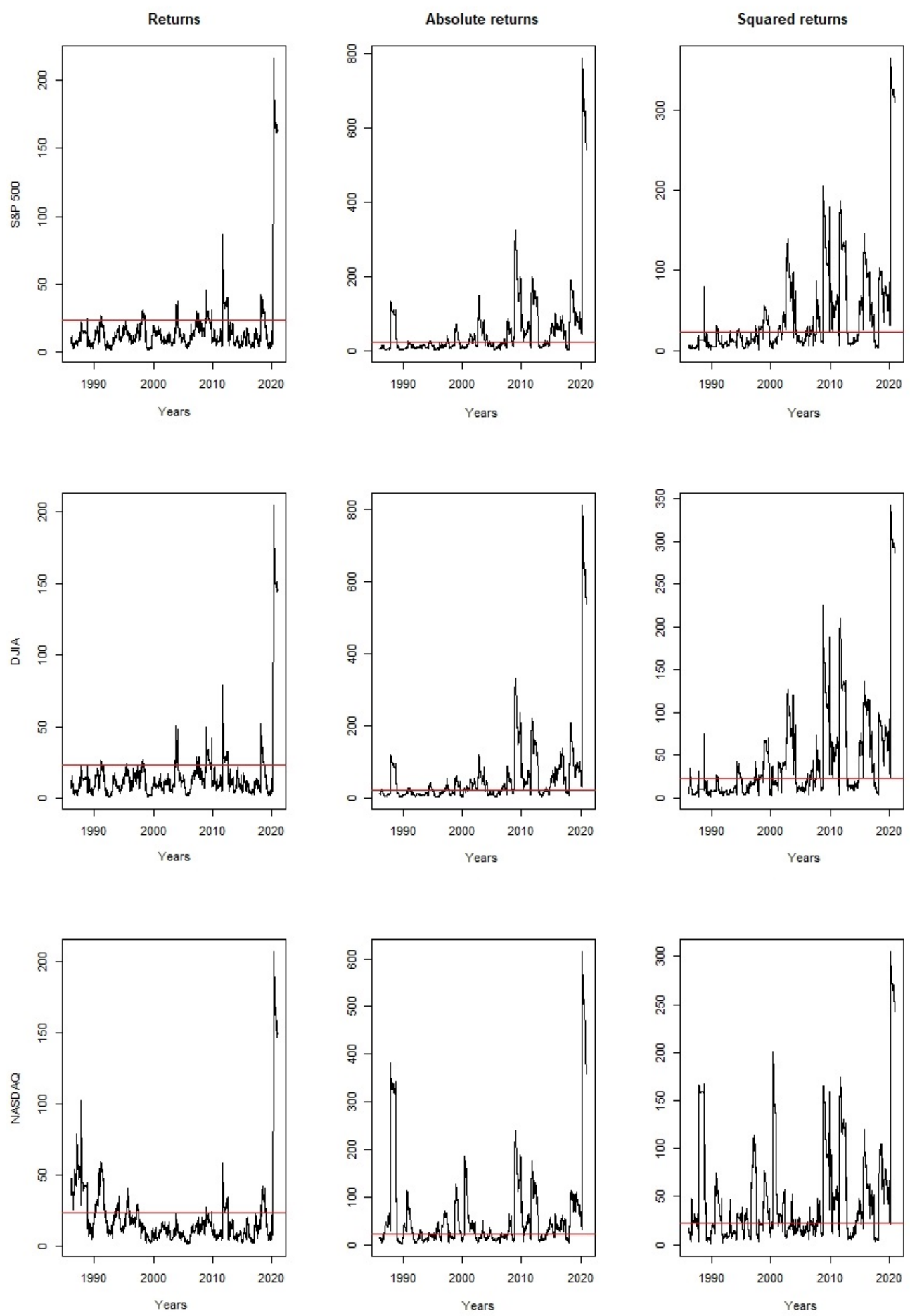
Fig. 11 Ljung-Box statistic - FATANG stocks
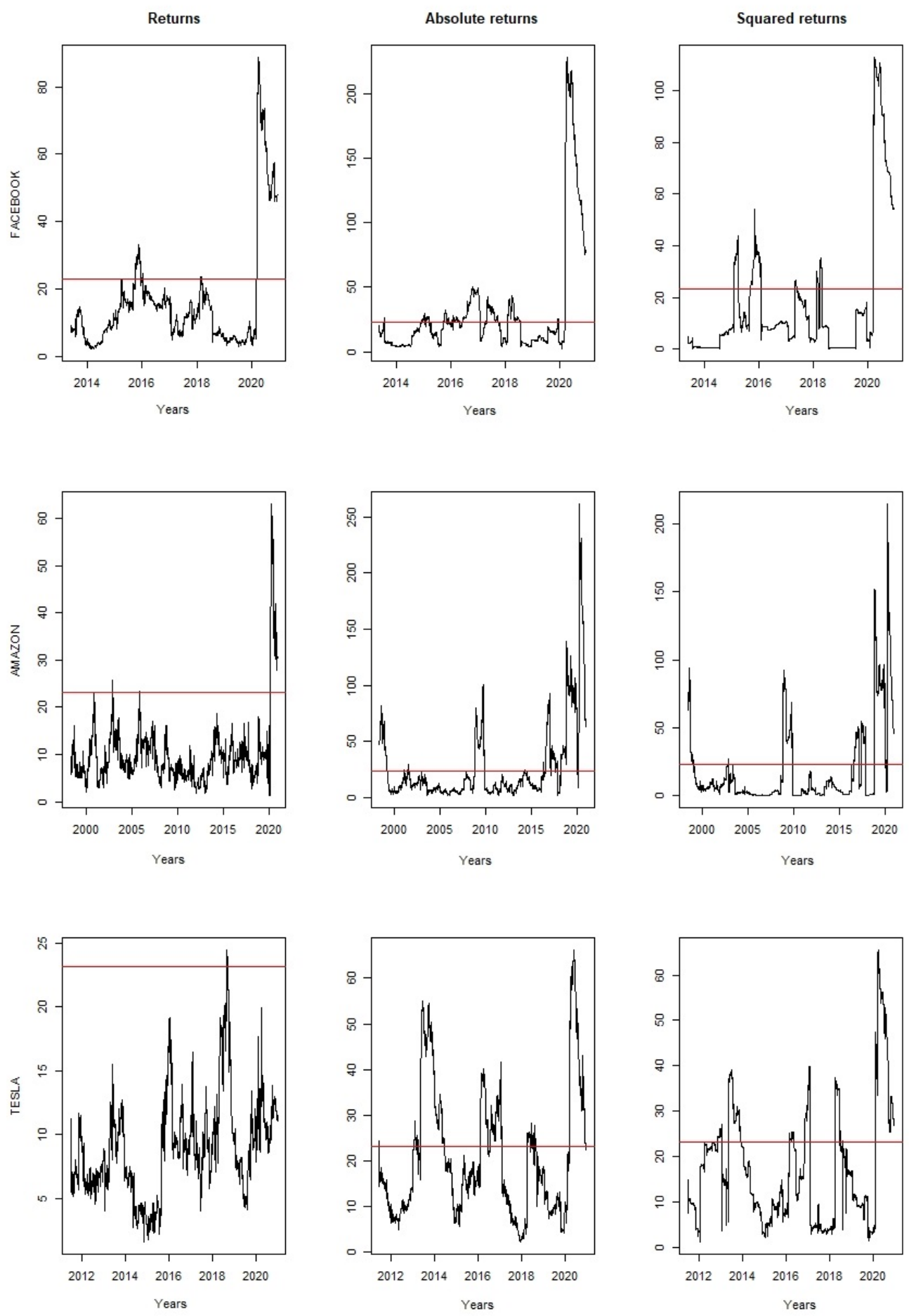
38

José Dias Curto

Fig. 12 Ljung-Box statistic - FATANG stocks
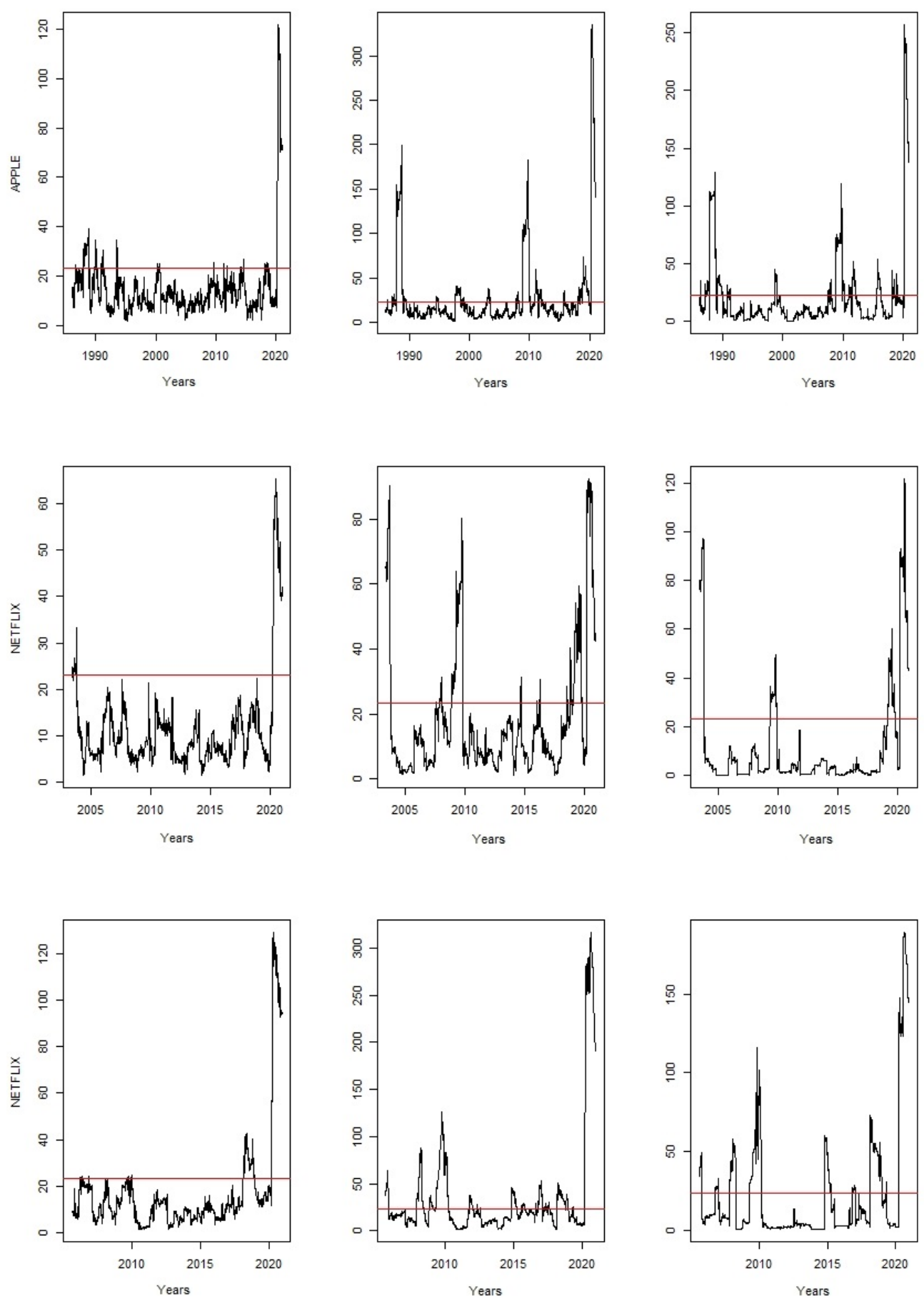
Fig. 13 ARCH LM statistic - US Indices
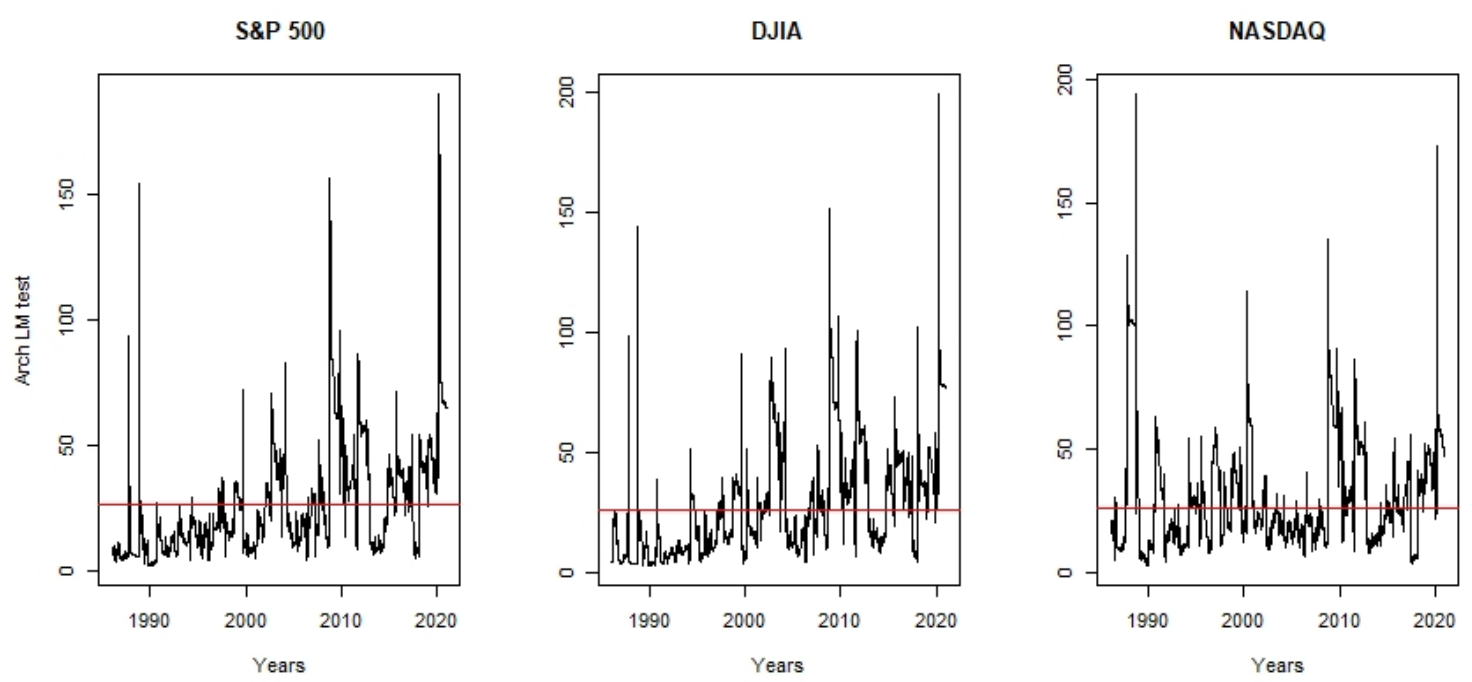
Fig. 14 ARCH LM statistic - FATANG stocks
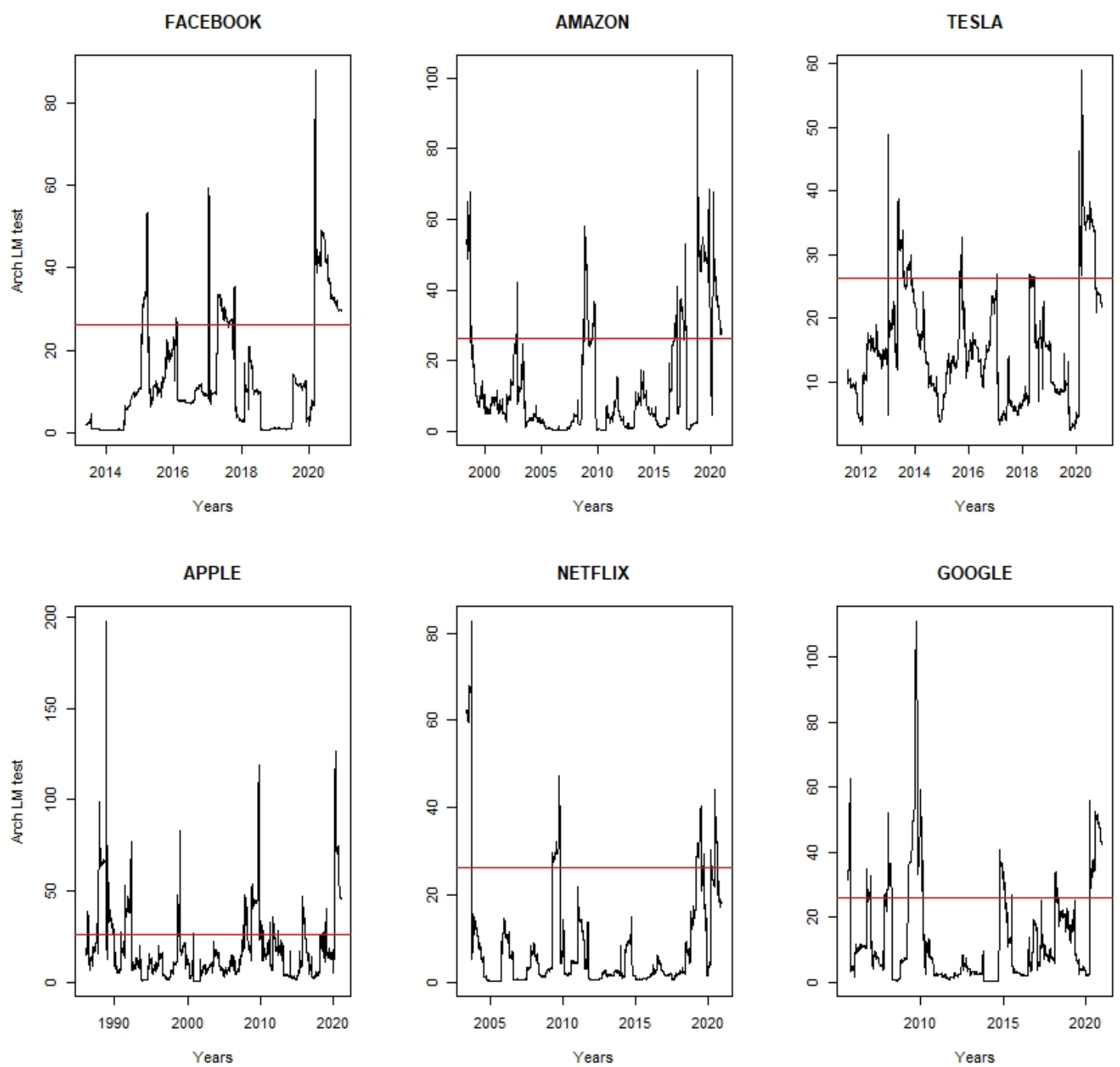
Figures

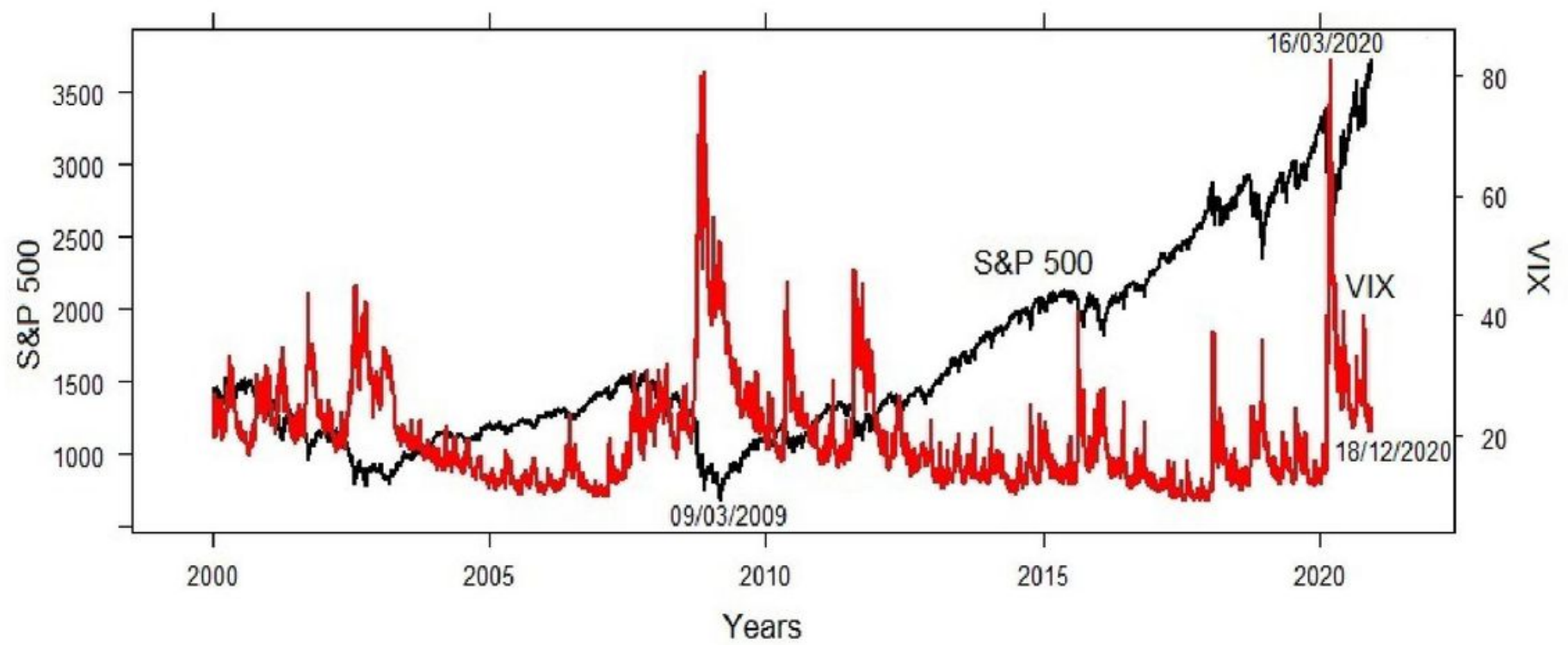

Figure 1

S\&P 500 and VIX
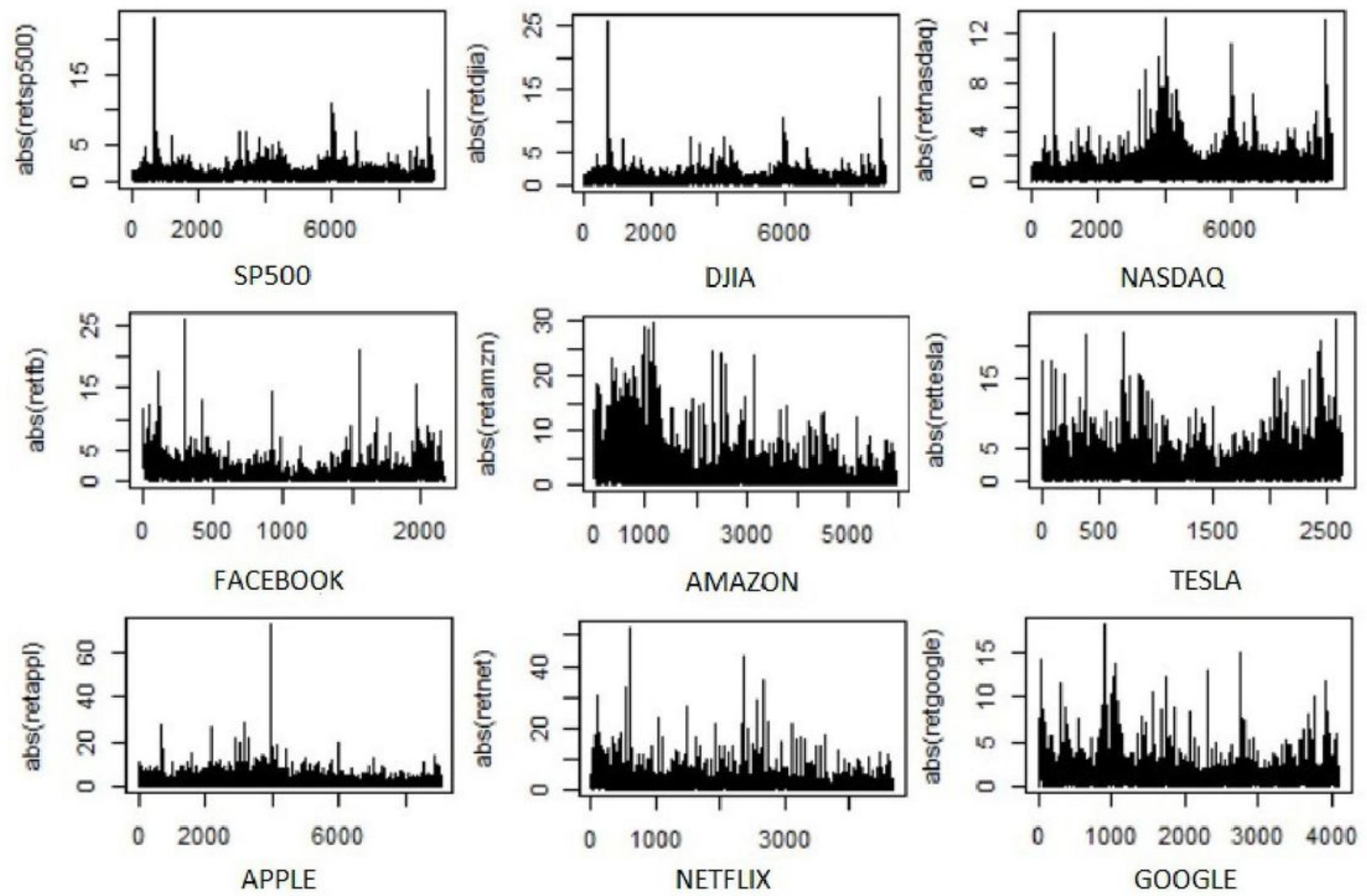

Figure 2 
Daily absolute returns
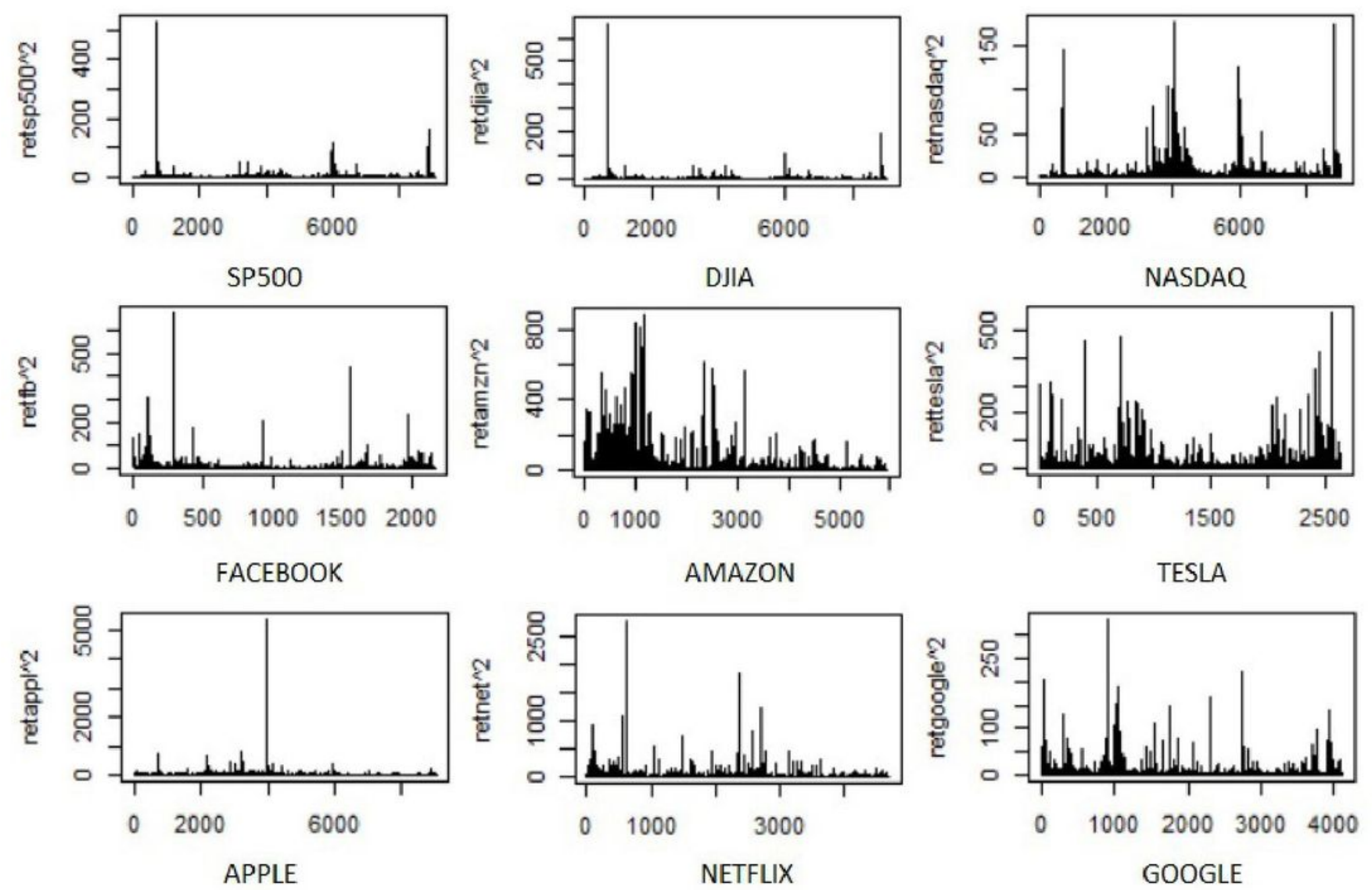

Figure 3

Daily squared returns 

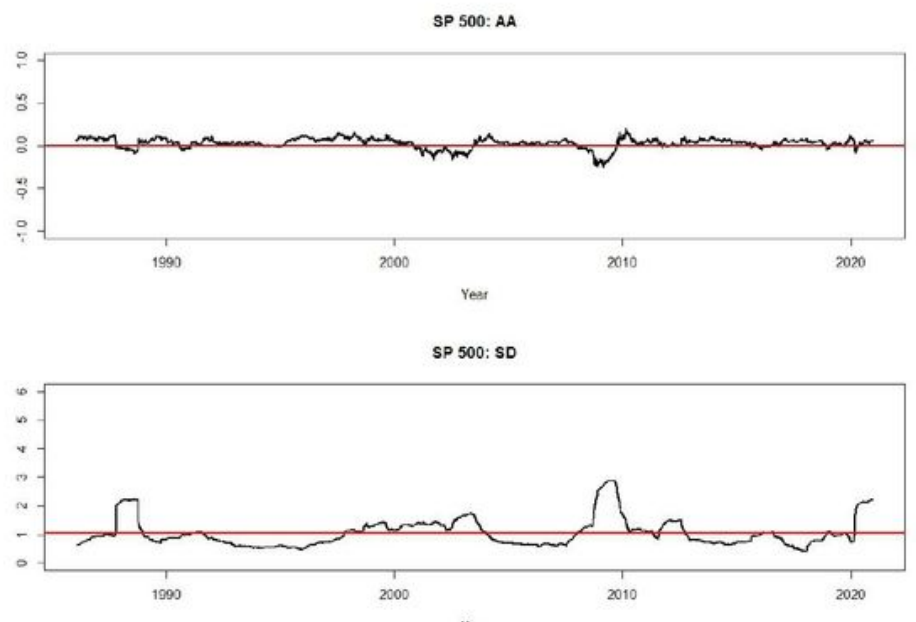

Year

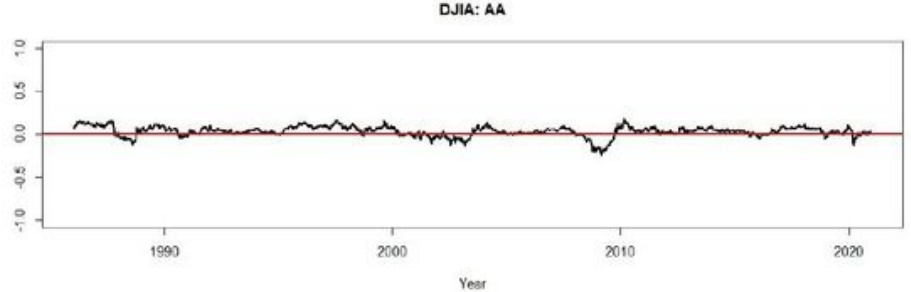

DJIA: SD

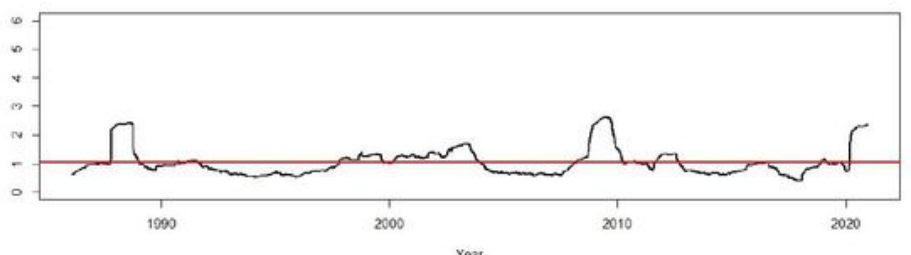

NASDAQ: AA

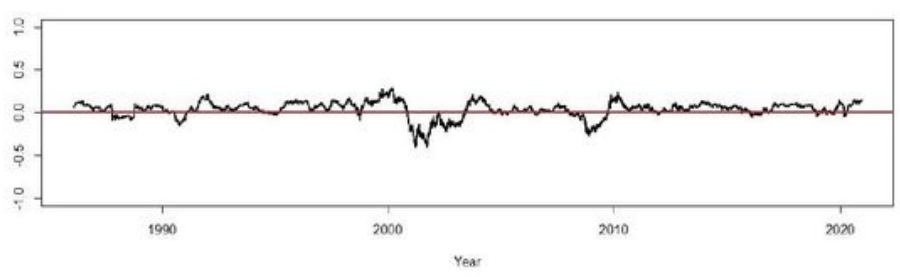

NASDAQ: SD

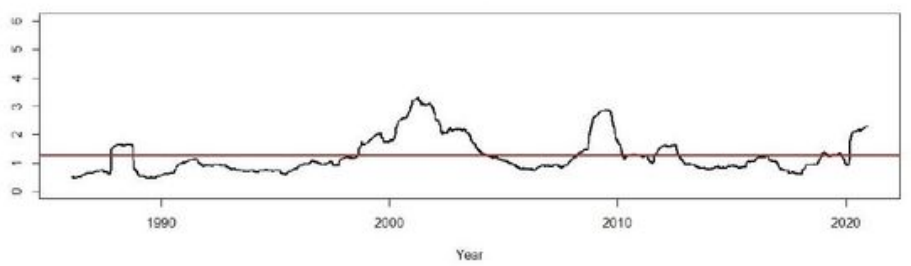

\section{Figure 4}

Rolling window mean (AA) and standard deviation (SD) - US Indices The Arithmetic Average (AA) and the Standard Deviation (SD) are computed over a rolling window encompassing the previous year of daily observations $(T=250)$. The horizontal lines represent the mean of each series. 

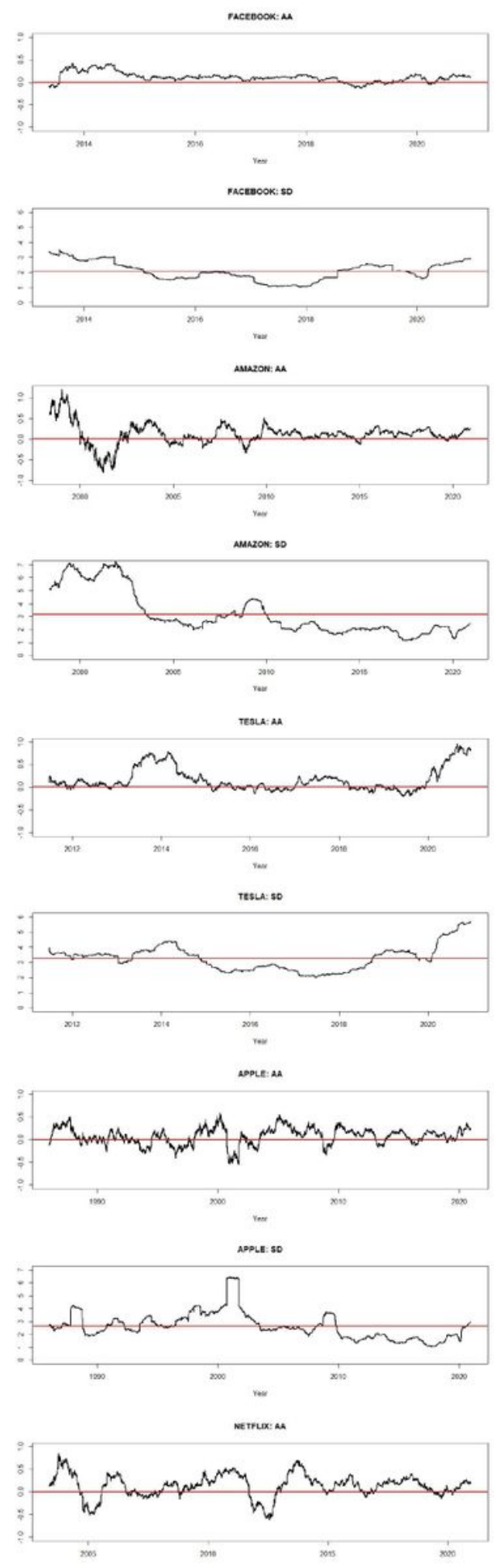

Figure 5

Rolling window mean (AA) and standard deviation (SD) - FATANG stocks 

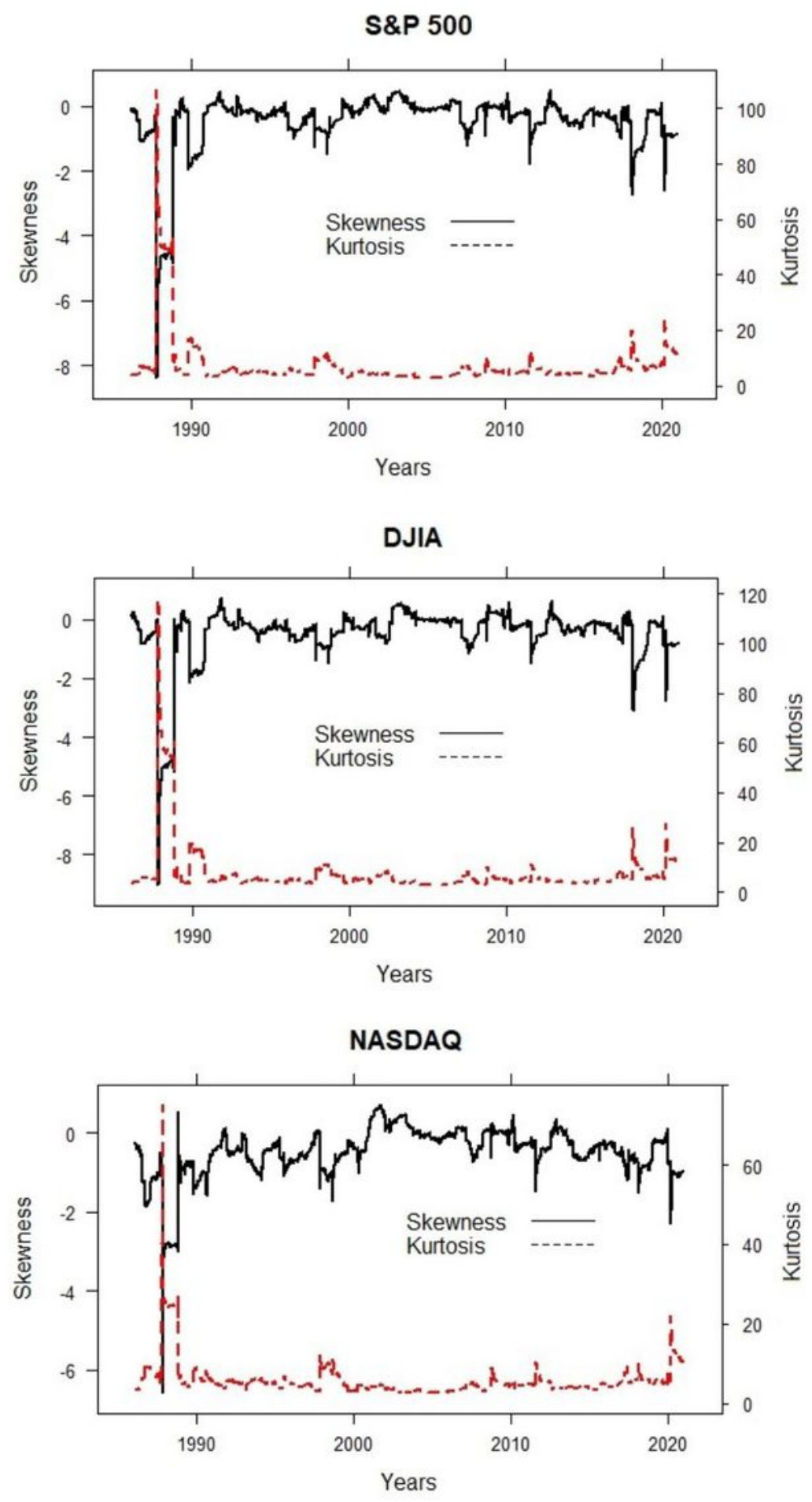

Figure 6

Skewness and kurtosis - US indices 

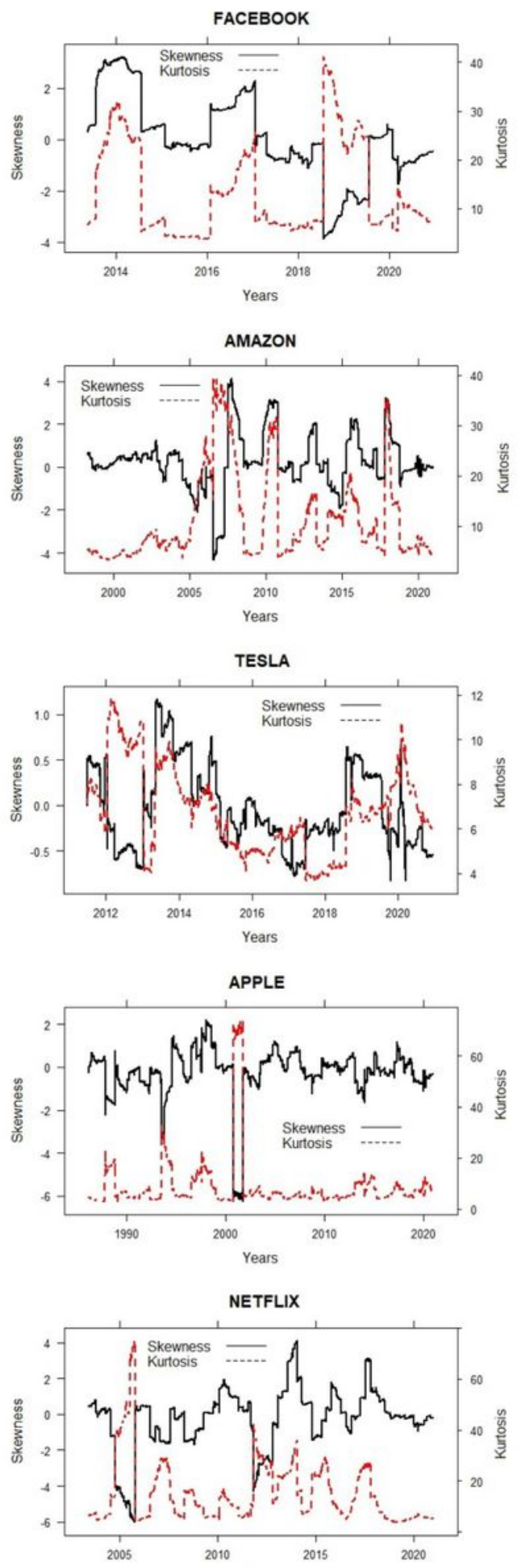

Figure 7

Skewness and kurtosis - FATANG stocks 

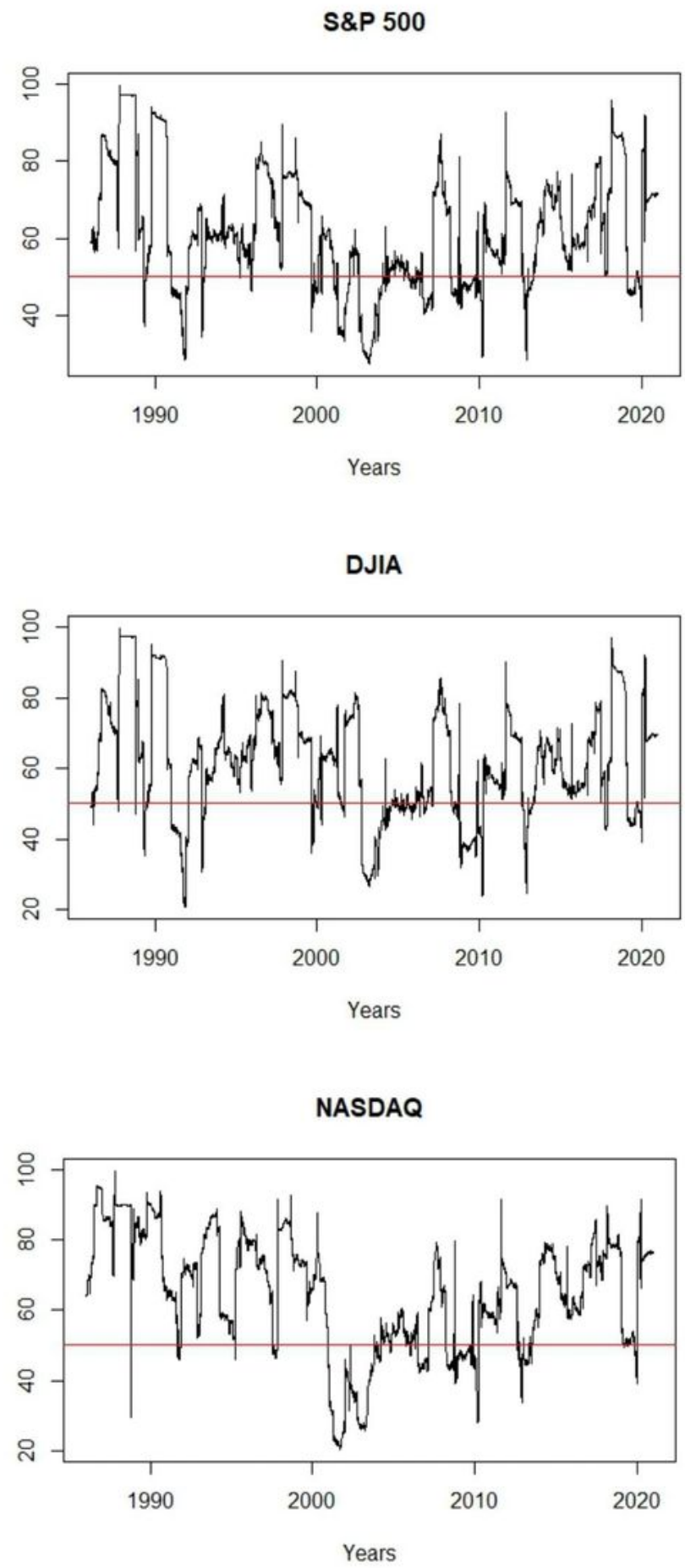

Figure 8

Ratio of the semi-kurtosis in the kurtosis - US Indices 

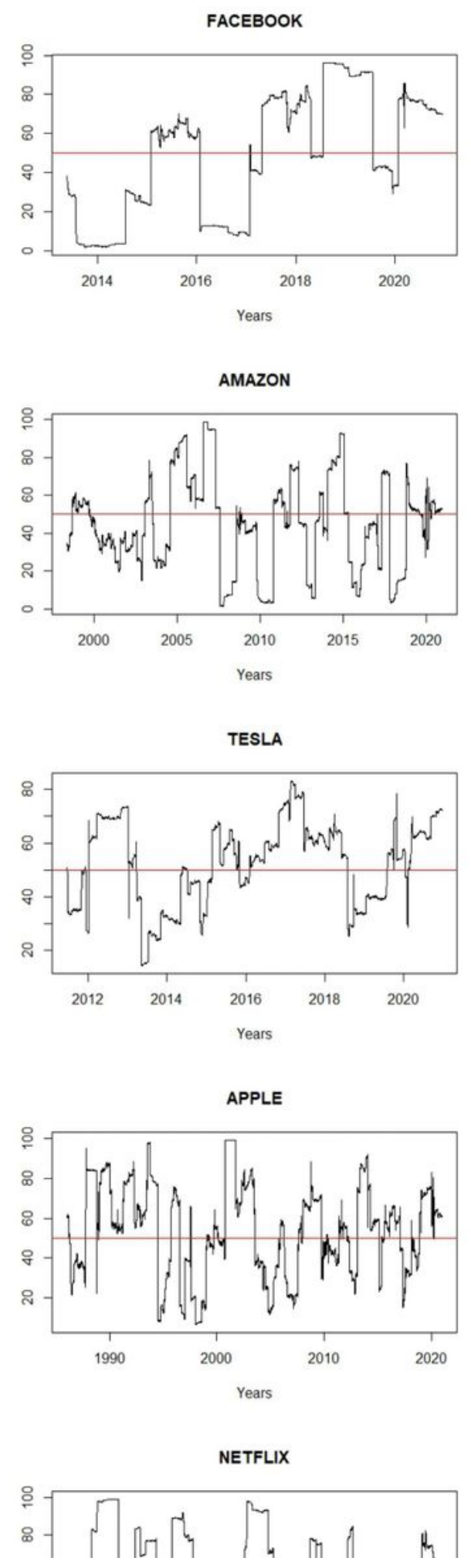

Figure 9

Ratio of the semi-kurtosis in the kurtosis - FATANG stocks 

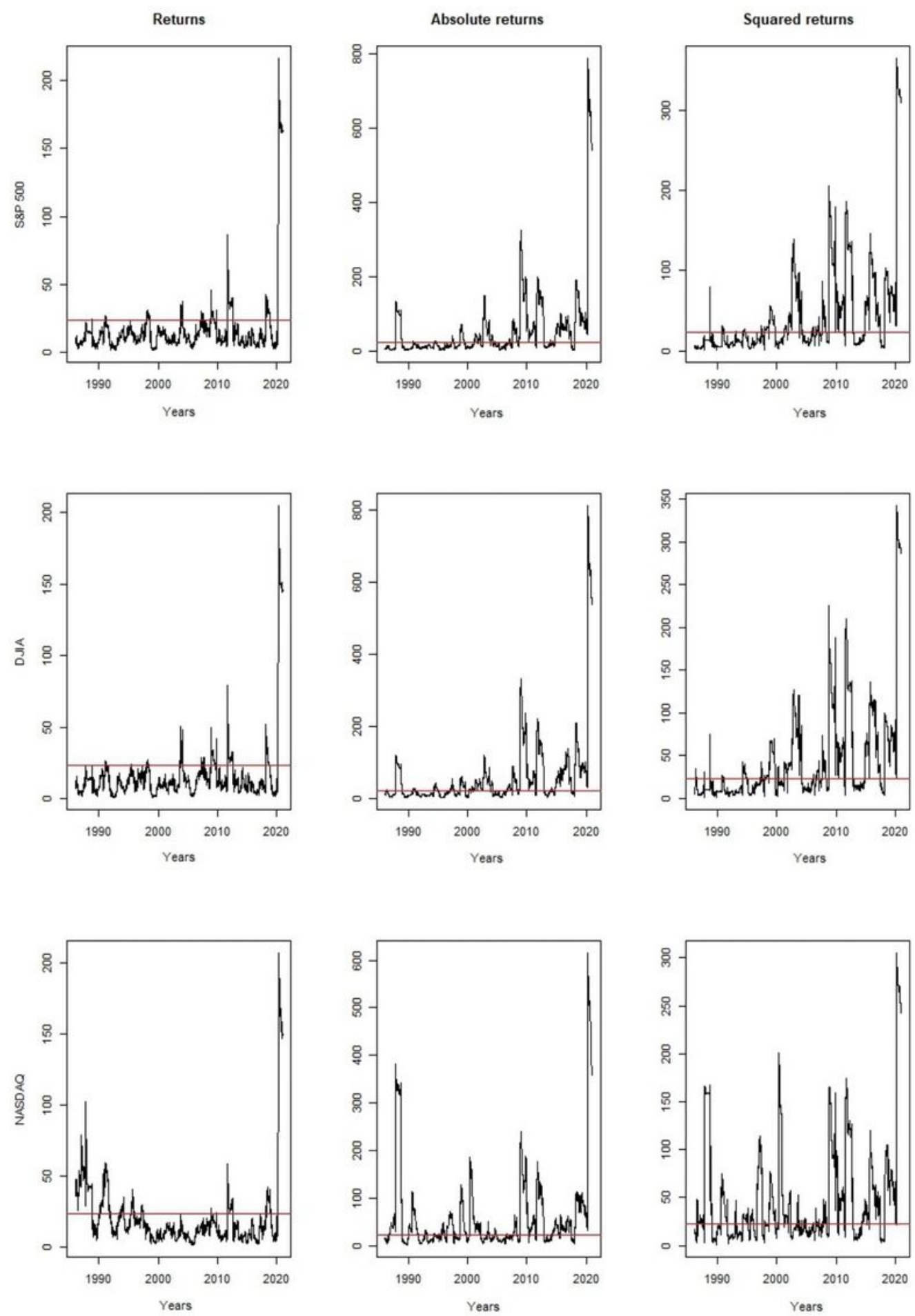

Figure 10

Ljung-Box statistic - US indices 

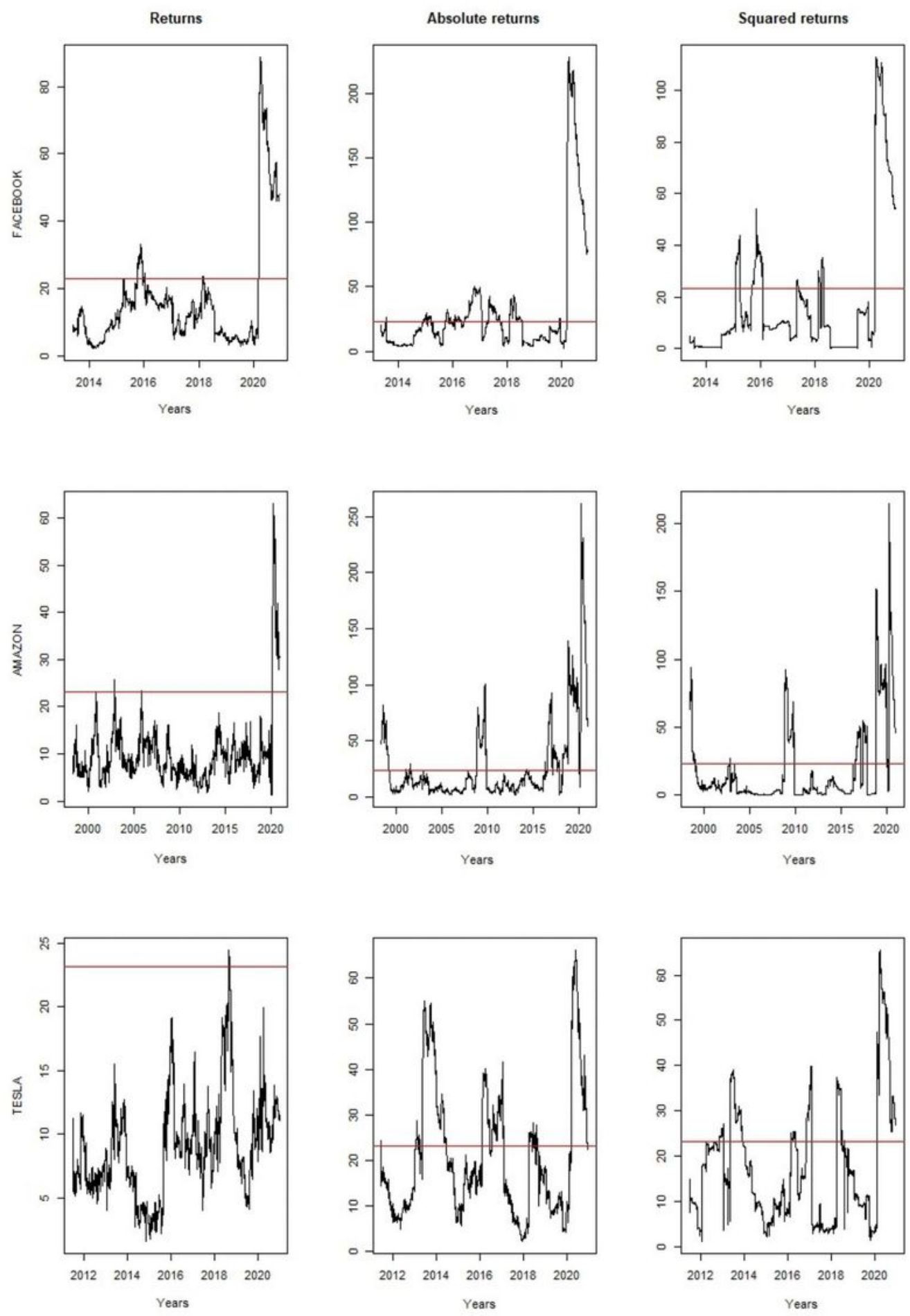

Figure 11

Ljung-Box statistic - FATANG stocks 

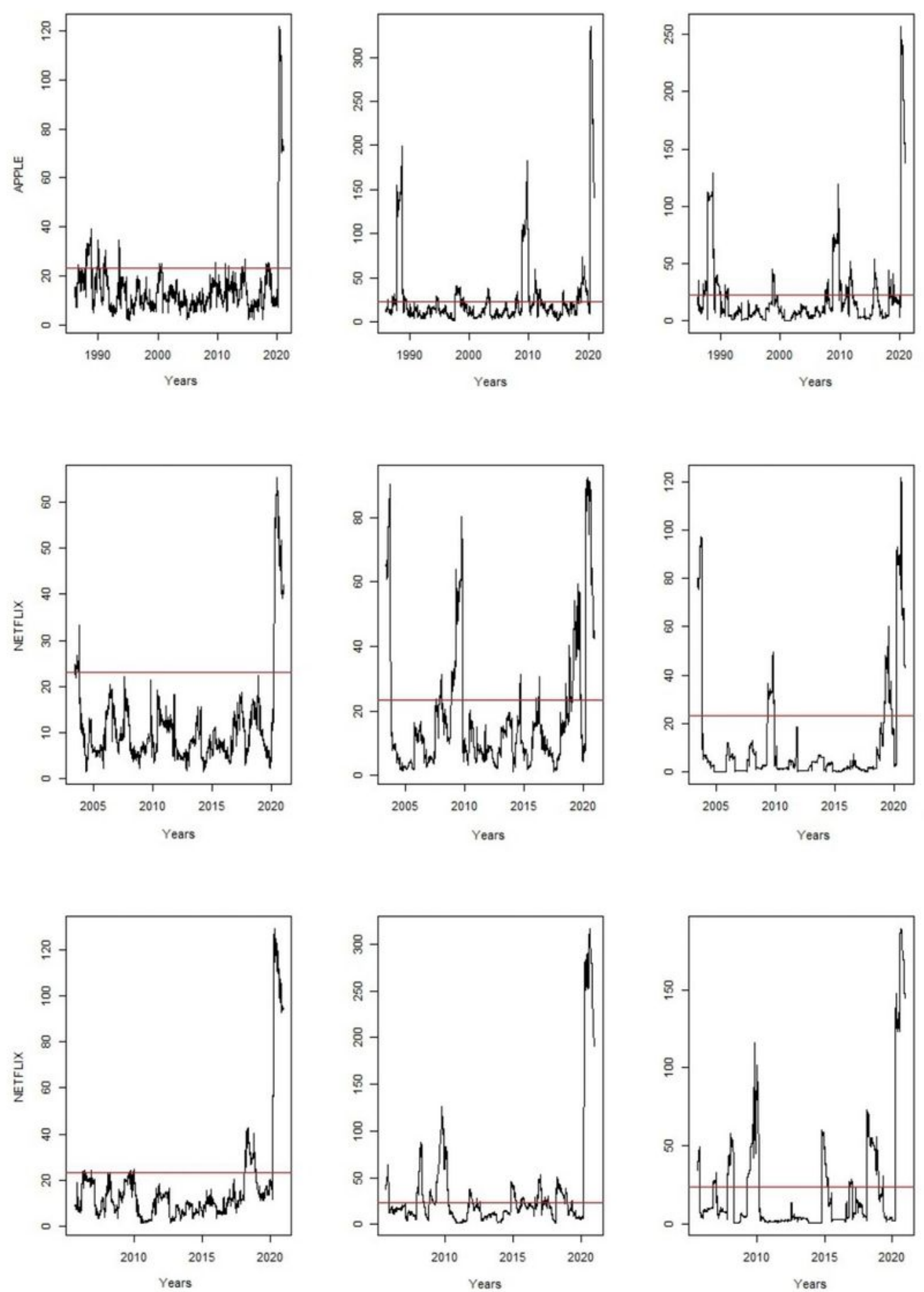

Figure 12

Ljung-Box statistic - FATANG stocks 

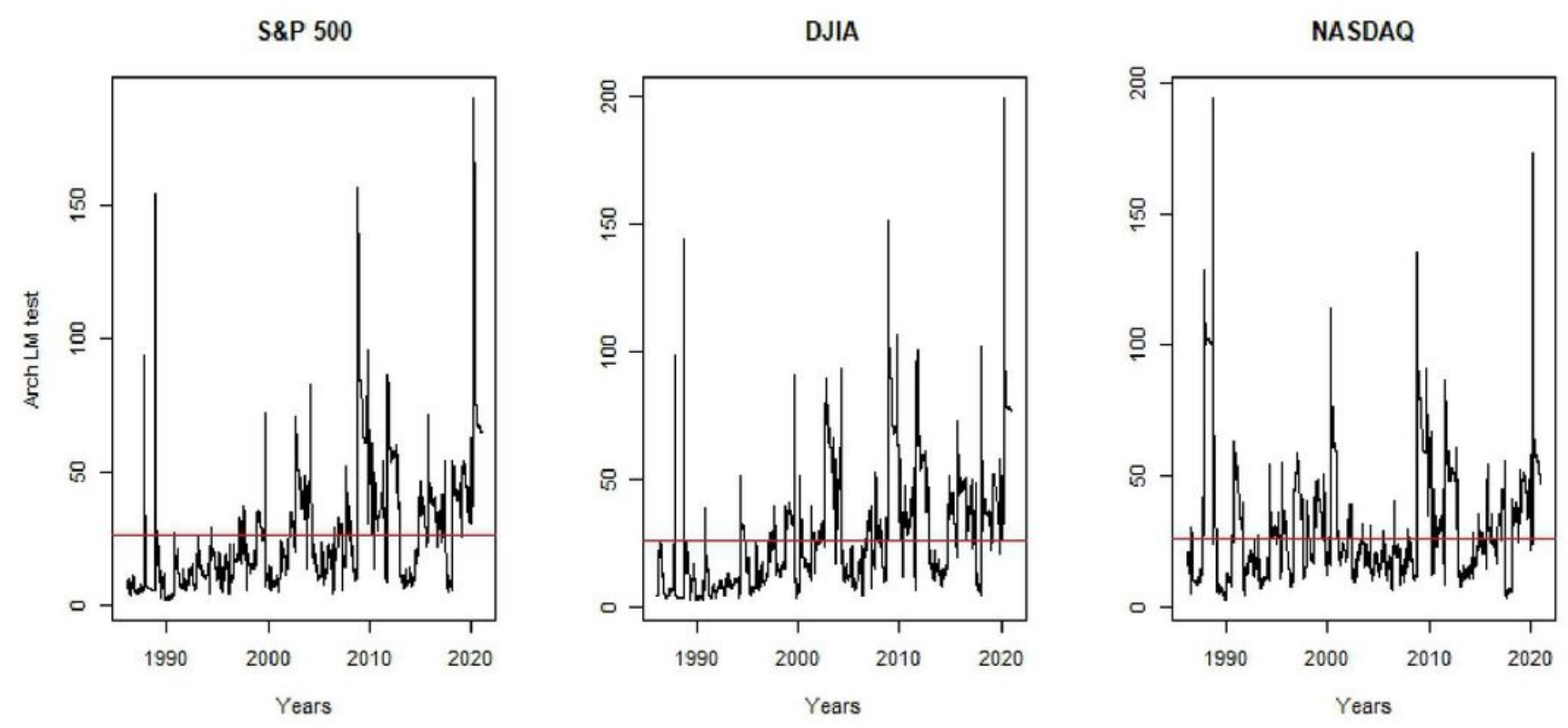

Figure 13

ARCH LM statistic - US Indices 

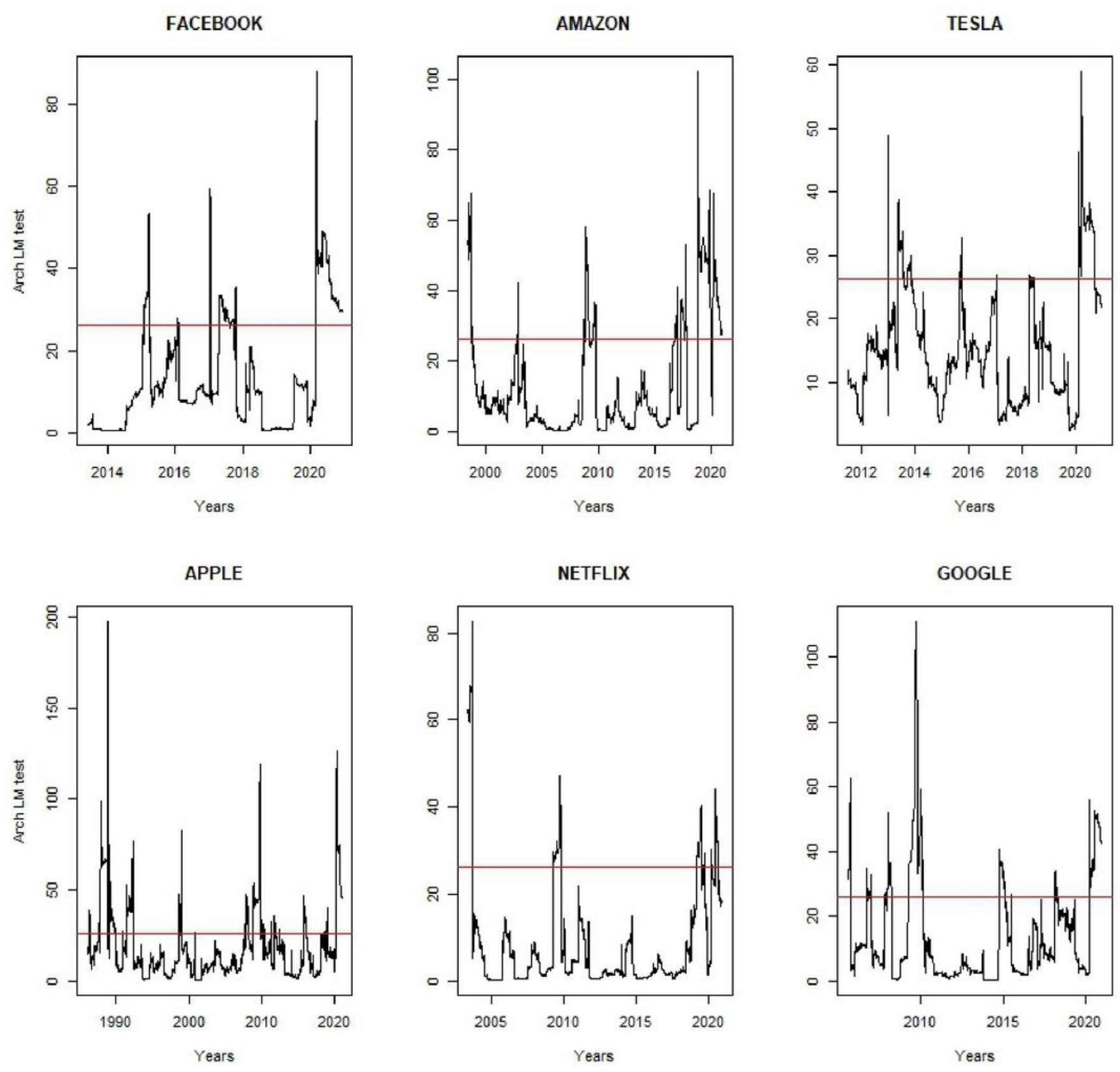

Figure 14

ARCH LM statistic - FATANG stocks 